\title{
Neural systems for memory-based value judgment and decision-making
}

Avinash R. Vaidya ${ }^{1}$

David Badre ${ }^{1,2}$

${ }^{1}$ Department of Cognitive, Linguistic, and Psychological Sciences, ${ }^{2}$ Carney Institute for Brain Science, Brown University, Providence, RI, 02912

ABBREVIATED TITLE: MEMORY-BASED VALUE JUDGMENT

\section{Address correspondence to:}

Avinash R. Vaidya

Brown University

Providence, RI 02912-1978

tel. 401-863-3634, fax 401-863-2255

email avinash vaidya@brown.edu

Conflicts of interest: The authors declare no competing conflicts of interest.

Acknowledgements: This work was supported by a Multidisciplinary University Research Initiative award from the Office of Naval Research (N00014-16-1-2832), an award from the James S. McDonnell Foundation, and a Ruth L. Kirschstein National Research Service Award to A.V. (NIMH F32 MH116592-01A1). We are grateful to Michael Frank for helpful comments and suggestions on the manuscript, as well as Apoorva Bhandari and Andra Geana for constructive discussion on methods and analysis. Thanks also to Adriane Spiro for help with data collection, and Henry Jones for assistance with behavioral piloting and analysis of normative data. 


\section{Abstract}

32 Real life choices often require that we draw inferences about the value of options based on

33 structured, schematic knowledge about their utility for our current goals. Other times, value

34 information may be retrieved directly from a specific prior experience with an option. In a

35 functional magnetic resonance imaging (fMRI) experiment, we investigated the neural

36 systems involved in retrieving and assessing information from different memory sources to

37 support value-based choice. Participants completed a task in which items could be conferred

38 positive or negative value based on schematic associations (i.e. schema value), or learned

39 directly from experience via deterministic feedback (i.e. experienced value). We found that

40 ventromedial prefrontal cortex (vmPFC) activity correlated with the influence of both

41 experience- and schema-based values on participants' decisions. Connectivity between

42 vmPFC and middle temporal cortex also tracked the inferred value of items based on

43 schematic associations on the first presentation of ingredients, prior to any feedback. In

44 contrast, the striatum responded to participants' willingness to bet on ingredients as a

45 function of the unsigned strength of their memory for those options' values. These results

46 argue that striatum and vmPFC play distinct roles in memory-based value judgment and

47 decision-making. Specifically, the vmPFC assesses the value of options based on information

48 inferred from schematic knowledge and retrieved from prior direct experience, while the

49 striatum controls a decision to act on options based on memory strength. 


\section{Introduction}

How can we predict the value of options without prior, explicit experience? For example, when building a table, a hammer has greater value than a ball of yarn. No previous experience using yarn in place of a hammer is needed to make this kind of value judgment. However, this evaluation does depend on knowledge about yarn, hammers and table construction. Structured knowledge about the world that can support this kind of inference is often referred to as a schema (Bartlett, 1932; Ghosh \& Gilboa, 2014; Piaget, 1952). Judging the value of options for accomplishing complex goals, like table building, requires inference based on these schematic representations.

Value does not always need to be inferred. In the same example, seeing a certain brand of varnish on display might remind you that your friend had recommended that varnish for your table last week. In this case, value relies on the retrieval of an explicit positive association with the item from a specific time and place. Here, value is a memorized feature of one or a few previous episodes, which may be brought to mind through mechanisms of episodic retrieval.

Relatively little is known about the neural systems involved in retrieving information from different memory sources to support value judgment. Lesion studies have shown that ventromedial prefrontal cortex (VmPFC) and orbitofrontal cortex (OFC) are necessary for decision-making when option values must be inferred from knowledge of task structure, but not when values can be retrieved from directly learned associations (Bradfield, Dezfouli, van

72 Holstein, Chieng, \& Balleine, 2015; Izquierdo, Suda, \& Murray, 2004; Jones et al., 2012; Reber

73 et al., 2017). It is unclear if these deficits arise because these regions represent task structure

74 (Schuck, Cai, Wilson, \& Niv, 2016; Wilson, Takahashi, Schoenbaum, \& Niv, 2014), guide 
75 retrieval based on these schemas (Eichenbaum, 2017), or specially utilize structured

76 knowledge during value judgment.

77 A parallel line of research has focused on the striatum at the intersection of goal-

78 directed retrieval and value-based decision-making. Recent work has argued that the

79 striatum is involved in assessing the value of information stored in memory and using this

80 information to bias cortical representations of choices with higher value (Shadlen \&

81 Shohamy, 2016). A mutually compatible alternative is that striatum integrates memory

82 signals into its basic decision making functions, including those related to memory itself

83 (Han, Huettel, Raposo, Adcock, \& Dobbins, 2010; Scimeca \& Badre, 2012), though these may

84 also be separable processes (Elward, Vilberg, \& Rugg, 2015).

85 The function of these regions in value judgment likely further depends on

86 coordination with several other regions for the retrieval of relevant information. Notably,

87 overlapping regions within the vmPFC and the striatum are connected with regions

88 canonically associated with episodic and schematic memory, and adaptive retrieval,

89 including the hippocampus (HPC), middle temporal cortex (MTC) and anterior ventrolateral

90 prefrontal cortex (aVLPFC) (Haber \& Knutson, 2010; Price, 2007). However, it is not known

91 how these regions interact to link retrieval to decision-making.

92 We tested three hypotheses from this prior literature on memory and value-based

93 decision making using a functional magnetic resonance imaging (fMRI) experiment: (1)

94 vmPFC is preferentially engaged when value judgments are based on structured knowledge

95 about the world (i.e. schema memory). (2) Striatum preferentially tracks experienced values,

96 in keeping with its role in both episodic memory and valuation. (3) vmPFC and striatum

97 interact with different memory stores in accord with their respective roles in constructing 
98 values through schema-based inference (vmPFC) versus direct, explicit experience 99 (striatum).

100 Methods

101 Behavioral Task and Experimental design

102 We investigated the neural systems involved in making value judgments based on

103 different memory sources using a novel experimental task. This task, and all other tasks not

104 administered through Amazon Mechanical Turk (AMT), were programmed in Psychtoolbox-

1053 (Brainard, 1997; Kleiner, Brainard, \& Pelli, 2007). In this experiment, participants took the

106 role of a restaurant chef choosing whether or not to serve ingredients to a customer (Figure

107 1). Each trial required participants to make a bet based on their belief that the customer

108 would like or dislike the displayed ingredient. Participants were instructed that if they

109 served an ingredient that the customer liked, they would receive 50 points, however if they

110 served an ingredient that the customer did not like, they would lose 25 points. If participants

111 chose not to serve an ingredient, they would receive 0 points. This asymmetry in the points

112 rewarded versus lost for betting trials was chosen to offset the overall loss aversion of

113 participants. In this task, loss aversion is expressed as a tendency to avoid endorsing

114 ingredients on the first presentation, as revealed in behavioral piloting.

115 After the participant made their choice, the customer's preference for a given

116 ingredient was always signaled by a cue above the correct response during feedback,

117 regardless of whether the participant chose to feed the customer or not, and all customer

118 preferences were deterministic. Thus, participants received complete information on each

119 trial regardless of their response. The feedback provided a source for learning the value of

120 an individual ingredient. If the participant did not respond in time, no information about the 
121 customer's preferences was given and participants were shown the word 'slow' in place of

122 feedback about points earned.

123 In addition to the information about ingredient preferences provided through

124 feedback, participants were told at the start of a block that the customer liked ingredients

125 pertaining to one recipe (positive recipe) and disliked another (negative recipe). In this way,

126 the participant could also infer the value of an ingredient on the basis of schematic

127 knowledge about what ingredients go with what recipes, without having received feedback.

128 For example, if the customer liked cake and disliked soup, flour might be positively valued

129 and onions might be negative by virtue of their association with each recipe. Participants

130 were instructed that these recipes were general and could encompass many potential

131 variants (e.g. the recipe 'tacos' might include both beef and fish tacos). The names of these

132 recipes were displayed at the top of the screen in each trial, with the positive recipe shown

133 in green and the negative recipe in red text.

134 At the start of each block, participants were told that they were about to feed a new

135 customer, and were given the names of the recipes that the customer liked and disliked. The

136 two recipes in each block were randomly selected without replacement from a set of six

137 recipes. The screen was then left blank for $12 \mathrm{~s}$ until the start of the first trial.

138 Participants were also instructed before the start of the task that each customer liked

139 and disliked other ingredients that were unrelated to these recipes (i.e. independent

140 ingredients'). The value related to these ingredients could not be anticipated in advance

141 based on their schematic associations, but rather would have to be acquired through

142 experience. 
144 ingredients, participants could retrieve a memory of previous feedback to make a decision.

145 All repeated presentations were separated by at least one other ingredient so that

146 participants were never making decisions about the same ingredient for multiple trials

147 successively.

148 Thus, the experiment had three factors by design: ingredient clustering (recipe or

149 independent), value (positive or negative) and presentation number $(1,2,3)$. For the

150 purposes of behavioral and fMRI analysis, any missed responses were not considered in the

151 count of ingredient presentations, as no value information was communicated to the

152 participant on these trials (e.g. if a participant missed the response deadline on the first

153 presentation, the second presentation would be considered the first, and the third

154 presentation would be considered the second in all analyses).

155 In each trial, participants saw an ingredient and were asked to respond by moving a

156 slider to either side of a bar to indicate their decision to serve an ingredient and their

157 confidence in that decision with a single response, similar to Scimeca, Katzman, and Badre

158 (2016). Moving the slider past the midway point to either side was considered a binary

159 selection of that side's response, while the distance of the slider from the midway point

160 provided a continuous index of confidence. The slider would start in a random position on

161 each trial and participants could then drag it toward the left or the right by holding down a

162 button on a fiber optic response pad (Current Designs, Philadelphia, PA) using their right

163 index or middle fingers. The slider would stop moving when it reached an end of the bar, or

164 when participants let the button go. Participants could not change the slider position after 
165 letting go of the button. The side of bar where participants placed the slider signaled their

166 choice, and the distance of the from the middle of the bar indicated their confidence.

167 Before the fMRI experiment, participants completed a practice task in which they

168 learned to use the slider while verifying various general knowledge statements (e.g., 'Black

169 bears are omnivores'), and indicate their confidence in their response. Participants received

170 further practice outside the scanner by completing a short practice block of the restaurant

171 task where they fed a customer with recipes and ingredients that did not appear in the fMRI

172 experiment. Before completing this practice block, all participants had to provide a detailed

173 verbal description of the task instructions, including the payoff structure, to the

174 experimenter. The experimenter verbally filled in any gaps in the participants' explanation

175 of the task before allowing participants to proceed into the practice phase.

176 Trials were preceded by a jittered inter-trial interval (ITI) drawn from a lognormal

177 distribution (0.5-6 s). In each trial, participants saw a food ingredient centrally displayed for

178 up to $2.7 \mathrm{~s}$, during which time they could choose whether or not to feed the ingredient to the

179 customer ('yes'/'no'). Stimulus presentations duration did not depend on response time. The

180 names of the recipes that each customer liked and disliked appeared at the top of the screen

181 on each trial, color coded to indicate the customer's preference.

182 After a response, there was a jittered inter-stimulus interval (ISI) drawn from a

183 uniform distribution (0.5-5.5 s). The remaining time from the response window and slider

184 movement period was also added to this interval. Participants then saw feedback about their

185 decision for $1 \mathrm{~s}$. A square above the correct answer would light up, and the amount of points

186 added or subtracted from participants' total would be displayed in this period. 
Trial-order, ITI and ISI lengths were drawn from sequences optimized for efficient estimation of the hemodynamic response across conditions of interest. These sequences

189 were optimized by generating 5000 random sequences, and using SPM12 to create event-

190 based design matrices for stimuli and feedback events in each sequence. The average

191 variance for each regressor explained by all other regressors in the matrix was calculated for

192 each sequence and multiplied by the total duration of the sequence to approximate design

193 efficiency. The three sequences with the lowest values in this measure (i.e. least covariance

194 in regressors with the shortest experiment time) were used for the fMRI experiment and

195 were randomly ordered for each subject. Participants completed three blocks of this task in

196 the scanner, each a separate fMRI run. Each block consisted of 84 trials total, with seven trials

197 for each value-clustering-presentation condition in each block (21 trials per condition total).

198 Participants

199 Twenty-six participants were recruited from the Providence, RI area to participate in

200 the fMRI experiment. Two participants were excluded due to movement greater than our

201 voxel size on at least one dimension during the functional scan, and one was excluded for

202 falling asleep during the experiment. Of the remaining 23 participants, four were male, their

203 mean age was 22.5 years $(S D=4.0)$, and their mean years of education was $15.5(S D=2.3)$.

204 All participants were right-handed, had normal or corrected-to-normal vision, and were

205 screened for the presence of psychiatric or neurological conditions, psychoactive medication

206 use, and contraindications for MRI. Two participants rejected all ingredients on the first

207 presentation of the positive independent condition, prohibiting analysis of their behavior or

208 ROI-level responses in a full 12-condition ANOVA after filtering for correct responses. One 
participant did not commit any errors on the second or third presentation of the positive recipe ingredients, prohibiting analysis of their error trials in this condition.

212 Mechanical Turk (AMT) to provide normative naming responses for images of food

213 ingredients. Four of these subjects were rejected for exceptionally low accuracy in this task

$214(<50 \%)$. The mean age of the remaining participants was 35.4 years (SD $=10.5)$, and 107 of

215 them were male.

A second sample of 221 participants were recruited from the United States through

217 AMT to provide normative data for ingredient-recipe associations. Twenty-nine of these

218 participants were rejected for failing to follow task instructions (most often by simply

219 writing the names of the ingredients rather than associated recipes). The remaining

220 participants had a mean age of 36.0 years (SD $=11.1)$, and 71 of them were male.

221 All participants across experimental and norming studies gave informed consent as

222 approved by the Human Research Protections Office at Brown University, and were

223 compensated for their participation. Participants in the fMRI experiment also received a

224 monetary bonus proportional to the points they earned in the main task.

225 Stimuli

Ingredient stimuli were obtained from the bank of standardized stimuli (BOSS; $\mathrm{N}=$

227 65) (Brodeur, Dionne-Dostie, Montreuil, \& Lepage, 2010; Brodeur, Guerard, \& Bouras, 2014),

228 food-pics database $(\mathrm{N}=50)$ (Blechert, Meule, Busch, \& Ohla, 2014), with a small set of

229 supplemental food images collected by A.V. from Google Images $(\mathrm{N}=4)$. These images were

230 selected to include basic food ingredients - i.e. items that might be used as parts of a recipe,

231 rather than completed meals. For example, an image of cheese would be included rather than 
232 an image of a pizza. All images were scaled to the same size and padded with white pixels or

233 cropped to fit the same area. Ingredients from the BOSS database were excluded if more than

$23420 \%$ of the normative sample either responded that they did not know the name of the

235 ingredient or did not recognize the ingredient. Ingredients were also rejected if the modal

236 name for the ingredient given by respondents was incorrect (indicating low recognition

237 accuracy), and if the H-value (a measure of naming disagreement across participants, similar

238 to entropy) was above 3.00.

239 Because similar naming data were not available for ingredients from food-pics or

240 Google Images, normative naming data were obtained through an AMT experiment.

241 Participants were shown ingredient images and asked to write the name of the ingredient in

242 a text box below, or write "I don't know." Responses that included common variants of

243 ingredient names were considered correct, while overly general names were marked as

244 incorrect (e.g. 'nuts' for an image of pecans). Ingredients where more than $20 \%$ of

245 participants did not know the name, or gave incorrect answers, were rejected. Naming

246 agreement was also examined by calculating an H-value for each ingredient; however, this

247 value did not exceed 3.00 for any ingredient in this set.

$248 \quad$ Normative recipe associations

249 Associations between ingredients and recipes were obtained through a norming

250 experiment conducted on AMT akin to single word generation paradigms used to derive

251 word associations (Postman \& Keppel, 1970). Participants were shown ingredient images

252 one at a time and were asked to write the name of the first recipe that came to mind using

253 the ingredient shown on screen. Unique recipe names were then extracted for each

254 ingredient and two researchers (A.V. and H.J.) manually processed these data. Alternative 
names, semantically similar responses and misspellings were all collapsed into single recipes, and ingredient specific information was removed to facilitate detection of overlap in

257 ingredient-recipe associations (e.g. responses like 'pumpkin soup' and 'mushroom soup'

258 were both reclassified as 'soup'). The end result of this process was a matrix with

259 endorsement counts for 102 ingredients in 1876 recipes. Six ingredients were then dropped

260 from this set, as they were visually too similar to other ingredients in the experiment, leaving

26196 ingredients total.

263 ingredient. As our task design required that each recipe have a large number of associate

264 ingredients (at least seven recipe ingredients, and some additional ingredients to be used as

265 independent items), we cycled through different parameters for filtering out recipes. With

266 each of these filtered lists, ingredients were assigned to hierarchically organized clusters

267 using the Matlab (Mathworks, Natick, MA) function 'linkage,' which sorted ingredients based

268 on the mean cosine similarity of their naming frequencies. Through this process we selected

269 six recipes where the clustering solution provided ingredient cluster sizes that were large

270 enough for our task design (pasta $(\mathrm{N}=10)$, soup $(\mathrm{N}=13)$, cake $(\mathrm{N}=15)$, pie $(\mathrm{N}=21)$, salad

$271 \quad(\mathrm{~N}=17)$ and sandwich $(\mathrm{N}=20))$.

272 Value rating task

273 At the start of the experimental session, participants completed a short rating task

274 where they were asked to provide ratings for how much they would hypothetically want to

275 have each ingredient at the end of the session. Participants provided responses on a seven

276 point Likert scale from -3 to 3, with labels on -3 reading 'Not at all,' 0 reading 'Indifferent' 
variable in each of the parametric GLM analyses, and used to test if participants' personal

279 preferences influenced their decisions in the task in model-based analyses. This task also

280 served to visually familiarize participants with all ingredient images.

281 Computational modelling of behavior

282 Computational models of behavior were used to characterize the dynamics

283 underlying participants' behavior in this task. We developed a simple base model that made

284 a set of assumptions about behavior in this task that were carried over to all alternative

285 models. Each alternative model made a modification of this base model, or the best model

286 from the last step. Alternative model modifications were assessed and compared using a

287 cross-validation procedure, described in detail below. For simplicity, we present the final

288 best fitting model that resulted from this process. We also briefly describe alternative model

289 modifications that did not survive the selection process in the following section.

290 Within the best fitting model, the value $(V)$ of the ingredient $(i)$ on each trial was

291 calculated as:

$$
V_{i}=\beta_{0}+\beta_{u}\left(1-\left|E V_{i}\right|\right)+\beta_{e} E V_{i}+\beta_{s} S V_{i}(\text { Eq. 1) }
$$

293 Where $\beta_{e}$ controls the influence of experienced values $(E V)$, and $\beta_{s}$ controls the influence of

294 schema values (SV) for the current trial ingredient (these two terms were included in the

295 base model). The parameter $\beta_{u}$ is a free temperature parameter that controls the model's

296 tendency to endorse or reject an ingredient based on its unfamiliarity (the opposite of the

297 absolute value of the experienced value term - described below; unfamiliarity model). An

298 intercept term, $\beta_{0}$ captures overall, item-independent biases toward betting or passing

299 (fixed bias). The model's willingness to bet on each trial (i.e. the model-P(yes)) was then

300 calculated by placing $V_{i}$ in a simple logistic function. 
On each trial where participants responded, the experienced value for each

302 ingredient was updated according to a simple Rescorla-Wagner learning rule (Rescorla \&

303 Wagner, 1972):

304

$$
E V_{i}=E V_{i}+\alpha\left(\text { outcome }-E V_{i}\right)(\text { Eq. 2) }
$$

305 Where the value for outcome could be 1 or -1 , depending on whether the feedback was

306 positive or negative. While this rule is more commonly used in the context of reinforcement

307 learning, it was adopted here as a simple means of operationalizing a graded memory that is

308 signed and becomes stronger with repetitions of consistent value associations. The

309 parameter $\alpha$ was a fixed learning rate that was fit for each subject in an alternate version of

310 the base model where $\beta_{e}$ was held at different constant values $(1,2,5$ and 8$)$. The model fit

311 with a constant value of 5 for $\beta_{e}$ had the lowest average negative log-likelihood of constants

312 tested, and so $\alpha$ values from this fit were used in the base model and all further variations.

313 This procedure to separately fit $\alpha$ was followed because $\beta_{e}$ and $\alpha$ could not be stably

314 estimated within the same model. Estimating these parameters separately allowed us to

315 roughly capture individual differences in the encoding of $E V$, while separately estimating the

316 contribution of $E V$ to choice behavior through $\beta_{e}$. Allowing $\alpha$ to vary was also found to

317 improve the fitness of the base model over a variant where $\alpha$ was fixed to 1.0.

To reflect forgetting, the values for all ingredients also decayed towards zero with

319 each trial according to:

$$
E V_{t+1}=E V_{t}-d E V_{t} \text { (Eq. 3) }
$$

321 Where $d$ is a parameter that controls the rate of this decay (also included in the base model). 
324 ingredient with these recipes was determined through a procedure based on latent semantic

325 analysis (LSA) (Dumais, 2004; Landauer, Foltz, \& Laham, 1998). The endorsement

326 frequencies for ingredients across all recipes in the normative AMT data were weighted by

327 their entropy across recipes and a singular value decomposition (SVD) was carried out on

328 this matrix. This matrix was then reconstituted with a smaller number of dimensions set by

329 a free parameter $c$ (dimensions model). This parameter essentially controlled the

330 dimensionality of recipe space that the model would consider, so that a lower dimensional

331 model would separate ingredients on only simple features (i.e. savory or sweet), while a

332 higher dimensional model would include increasingly granular information that separates

333 ingredients along information about specific recipes (Figure 2). Notably, allowing this

334 parameter to vary did not dramatically influence the overall choice behavior of the model in

335 simulations, but did change the shape of schema values so that increased dimensionality

336 increased the discretization within this term. The association of an ingredient with each

337 recipe was calculated as the mean cosine similarity between that ingredient and all

338 ingredients in each recipe cluster within this reconstituted matrix. The schema value for

339 each ingredient was then calculated as the difference in the associative strength for the

340 positive and negative recipe.

341 In subsequent presentations, the model discounted schema values with an

342 exponential function based on memory strength for experienced values (i.e. the absolute

343 value of $E V$ ):

344

$$
\text { discount }=\exp \left(-k\left|E V_{i}\right|\right)(\text { Eq. } 4)
$$

345 Where $k$ was a free parameter that determined the steepness of this discounting. This

346 discount factor was then included in the calculation of ingredient values: 


$$
V_{i}=\beta_{e} E V_{i}+\operatorname{discount} \beta_{s} S V_{i} \text { (Eq. 5) }
$$

348 So that the contribution of schema values scaled with this exponential discounting factor.

349 Alternate model parameters

350 Briefly, the following alternate modifications to the base model were considered, but

351 did not improve model fitness:

352 (1) $\underline{\text { Asymmetric schemas model }}$

353 This model included separate temperature parameters for positive and negative

354 schema values, so that the value of an ingredient was:

$$
V_{i}=\beta_{e} E V_{i}+\beta_{s p} P R_{i}-\beta_{s n} N R_{i}(\text { Eq. 6) }
$$

356 Where $\beta_{s p}$ and $\beta_{s n}$ are temperature parameters that control the influence of ingredient

357 associations with positive and negative recipes, respectively.

358 (2) Schema similarity model

The recipes in a block could vary in their similarity, making some ingredients

360 plausibly associated with both positive or negative recipes (e.g. pie and cake). To

361 accommodate this possibility, we tested a model where the associative strength of

362 ingredients with the positive and negative schemas to the schema was weighted by an

363 estimate of participants' uncertainty about the recipe associations of each ingredient,

364 calculated as the probability that an ingredient was associated with both recipes (schema

365 similarity model; $P(S R)$ ):

366

$$
P(S R)_{i}=\frac{1}{1+\exp \left(-\beta_{\operatorname{sim}}\left(1-\frac{\left|P R_{i}-N R_{i}\right|}{R R D}\right)\right.} \text { (Eq. 7) }
$$

367 Where $R R D$ corresponds to the range of recipe association differences (i.e. the difference

368 between the maximum and minimum recipe associative strength for all ingredients and 
recipes). This probability was then used in calculating weights for the sum and difference of

370 the positive and negative recipe associations, where the weight for the sum of these

371 associations was calculated as

$$
w_{\text {same }}=2\left(P(S R)_{i}-0.5\right)(\text { Eq. } 8)
$$

373 And the weight for the difference of these ingredient associations was calculated as

$$
w_{\text {diff }}=1-2\left|P(S R)_{i}-0.5\right|(\text { Eq. 9) }
$$

$375 S$ was then calculated as the weighted combination of the difference and sum of each

376 ingredient's associative strength with the positive and negative recipes:

$$
S V_{i}=w_{\text {same }}\left(P R_{i}+N R_{i}\right)+w_{\text {diff }}\left(P R_{i}-N R_{i}\right)(\text { Eq. 10) }
$$

378 The parameter $\beta_{\text {sim }}$ controlled sensitivity to the similarity of ingredients' recipe

379 associations, and the relative contribution of the difference or sum of positive and recipe

380 associations to $S V$. This parameter also controlled the valence of the additive strength of

381 these associations: positive or negative values for $\beta_{\text {sim }}$ would give the same valence to the

382 additive strength of recipe associations, respectively. Alternatively, if the $\beta_{\text {sim }}$ parameter was

383 zero, participants would rely solely on the difference of the ingredient's associative strength

384 with the positive and negative recipes.

385 (3) Dual process recollection model

386 While the above models assume that experienced values are stored in episodic

387 memory via a graded process, other models developed in memory judgment tasks suggest

388 that episodic memory has a graded component (familiarity) and a discrete component

389 (recollection) (Yonelinas, 1994). To test whether experienced values in this task may also

390 rely on an additional non-graded recollection process, ingredient values were calculated as:

$$
V_{i}=\beta_{e}\left(\left(1-P_{r}\right) E V_{i}+P_{r} R V_{i}\right)+\beta_{s} S V_{i} \text { (Eq. 11) }
$$


392 Where $R V$ is the recollected value of an ingredient, i.e. the outcome from previous trials that

393 does not decay or change over time ( -1 or 1$)$, and $P_{r}$ is a fixed probability of recollection that

394 does not change over trials. Thus, in this model, values learned through feedback contributed

395 to the decision as the sum of recalled and graded value information, weighted by the

396 probability of recall.

397 (4) Schema spreading model

398 Rather than treat experienced and schema-based values separately, it is possible that

399 participants may have used schematic knowledge to try and facilitate feedback-based

400 learning. To test this hypothesis, we created a model where values learned through feedback

401 were broadcast to other ingredients, weighted by their similarity in recipe space. Upon

402 feedback, the experienced value for ingredient $i$ was used to update a schema spread value

403 term (SSV) for all other ingredients ( $j$ ) according to:

404

$$
S S V_{j}=S S V_{j}+\left(1-\cos \left(\text { Ingredient }_{i}, \text { Ingredient }_{j}\right)\right) \alpha\left(\text { outcome }- \text { SSV }_{j}\right)(\text { Eq. 12) }
$$

405 Where $\alpha$ is the same learning rate used for updating experienced values and cos refers to 406 the cosine distance between ingredients in the normative ingredient-recipe association 407 matrix produced through LSA.

409 weighted by an additional free temperature parameter.

410 (5) Participant subjective value

411 We also tested an alternate model that included participants' own subjective value

412 ratings for ingredients in the computation of ingredient values weighted by an additional

413 free temperature parameter. This model tested whether participants' choices would be

414 better accounted for by taking into account their personal preferences. 
Model fitting and comparison

417 whether or not to feed an ingredient to a customer, and a continuous rating of their

418 confidence in this binary response. This confidence response could also be transformed into

419 a continuous measure of participants' belief that a customer would like an ingredient (i.e.

$420 \mathrm{P}(\mathrm{yes})$ ) by measuring the position of the slider between the 'yes' and 'no' options rather than

421 the distance of the slider from the middle, similar to Scimeca et al. (2016). Models were fit to

422 both participants' continuous and binary choice responses. The overall model likelihood was

423 calculated as the product of the model likelihood for participants' binary responses, and the

424 model likelihood of the linear fit between the logit transformed model-P(yes) and

425 participant-P(yes). The negative log of this likelihood function served as the objective

426 function to be minimized in the fitting procedure carried out by the Matlab function

427 'fmincon.'

429 each step, a simpler preferred model was modified by the addition of new free parameters.

430 Models were compared with a cross-validation procedure. For each participant, we

431 generated 100 independent training and test datasets where a set of 16 ingredients (19\% of

432 the total ingredients) were randomly held out of the data used for fitting model parameters

433 and treated as deadline failures (as to maintain the same number of trials in the experiment).

434 We chose to randomly subsample ingredients rather than blocks or trials because of the

435 structure of the task: participants encounter a new customer, and thus a new set of recipe

436 schemas in each block, while trials are not independent from each other, as each ingredient

437 is repeated three times and the value must be retained from one trial to the next. 
To test model fitness, we estimated the mean difference in the negative log-likelihood

439 of each model relative to the base model for the held out 16 ingredients, averaged across all

440100 test datasets and summed across participants. At each step in the model comparison

441 process, the model that resulted in the largest reduction in the negative log-likelihood for the

442 test data set was considered the preferred model. Additional parameters reduced the

443 negative log-likelihood were added to the preferred model and compared in the next step,

444 while any parameters that did not improve model fitness were discarded in future steps. The

445 search for the best fitting model was stopped once additional parameters no longer

446 improved model fitness, or when the parameters to search across were exhausted.

447 Each model was fit separately for each subject 20 times with randomized starting

448 positions. The set of parameters with the lowest negative log-likelihood from each set of fits

449 was saved and the other parameter fits were discarded. These 20 separate fits helped guard

450 against the influence of idiosyncratic differences in starting point on the recovered

451 parameters, and the possibility that the model fitting process would converge on local

452 minima in the negative log-likelihood function.

453 Parameter recovery test

454 To verify that all parameters of the best fitting model could be recovered, we 455 generated 100 simulated datasets with randomized parameters. The parameter $d$ was 456 generated from a uniform distribution from 0 to 0.05 . Parameters $\beta_{e}$ and $\beta_{s}$ were generated

457 from a lognormal distribution with a mean of 5 and standard deviation of 0.5. Parameters

$458 \beta_{0}, \beta_{m}$ and $\beta_{\text {sim }}$ were all generated from a standard normal distribution (mean 0 , standard

459 deviation of 1 ). The $c$ parameter was generated from a uniform distribution ranging from 2

460 to 58. Given that these parameters were randomly generated and did not share the 
461 covariance of the real parameters fit to participants' behavior, this test could overestimate

462 their recoverability. Thus, we further verified the recoverability of these parameters by

463 fitting the model to data simulated using the parameters fit to participants' real behavior.

464 fMRI data acquisition and preprocessing

465 Whole-brain imaging was acquired using a Siemens 3T Magnetom Prisma system

466 with a 64-channel head coil. First, a high resolution T1 weighted MPRAGE image was

467 acquired for visualization (repetition time (TR), 1900 ms; echo time (TE), 3.02 ms; flip angle,

$46899^{\circ} ; 160$ sagittal slices; $1 \times 1 \times 1 \mathrm{~mm}$ voxels). Functional volumes were acquired using a

469 gradient-echo echo planar sequence (TR, $2000 \mathrm{~ms}$; TE, $28 \mathrm{~ms}$; flip angle $90^{\circ}$; 38 interleaved

470 axial slices tilted approximately $15^{\circ}$ from the AC-PC plane; $3 \times 3 \times 3 \mathrm{~mm}$ voxels). Functional

471 data were acquired over three runs. Each run lasted 16.5 min on average (Mean: 494.8

472 acquisitions). After the functional runs, a brief in-plane anatomical T1 image was collected,

473 which was used to define a brain mask that respected the contours of the brain and the space

474 of the functional runs (TR, $350 \mathrm{~ms}$; TE $2.5 \mathrm{~ms}$; flip angle $70^{\circ} ; 38$ axial slices; $1.5 \times 1.5 \times 3 \mathrm{~mm}$

475 voxels). Soft padding was used to restrict head motion throughout the experiment. Stimuli

476 were presented on a 32-inch monitor at the back of the bore of the magnet, and subjects

477 viewed the screen through a mirror attached to the head coil.

478 Functional data were preprocessed using SPM12. Quality assurance for the functional

479 data of each subject was first assessed through visual inspection and TSdiffAna

480 (https://sourceforge.net/projects/spmtools/) ArtRepair

481 (http://cibsr.stanford.edu/tools/human-brain-project/artrepair-software.html). Outlier

482 volumes (defined by standard deviations from the global average signal) were interpolated

483 after preprocessing. Slice timing correction was carried out by resampling all slices to match 
484 the first slice. Next, motion during functional runs was corrected by registering volumes to

485 the mean using rigid-body transformation. In any case where movement across runs was in

486 excess of the voxel size in any direction ( $3 \mathrm{~mm})$, realignment was recalculated run-by-run.

487 Any participants where motion within a run was larger than a voxel were excluded from

488 further analysis $(\mathrm{N}=2)$. Functional volumes were then normalized to Montreal Neurological

489 Institute (MNI) stereotaxic space using $4^{\text {th }}$ order B-spline interpolation, and resampled to 2

$490 \times 2 \times 2$ mm voxels using trilinear interpolation. Finally, volumes were spatially smoothed

491 using an $8 \mathrm{~mm}$ full-width half-maximum isotropic Gaussian kernel. The in-plane anatomical

492 T1 image for each subject was normalized to MNI space and used to create a brain mask for

493 functional analysis using the Brain Extraction Tool in FSL

494 (https://fsl.fmrib.ox.ac.uk/fsl/fslwiki/).

495 fMRI data analysis

496 Functional data were analyzed under the assumptions of the GLM using SPM12. All

497 regressors and parametric modulators were convolved with the SPM canonical

498 hemodynamic response function (HRF). Subject motion (six translational and rotational

499 components) were also included as nuisance regressors. Beta coefficients for single subject

500 effects were estimated using a fixed-effects model in a first-level analysis. For whole-brain

501 contrasts, these estimates were then included in a second-level analysis using a one-sample

502 t-test against 0 at each voxel. Whole brain t-statistic maps were thresholded at a cluster

503 defining threshold of $P<0.001$ uncorrected, and then evaluated for statistical significance at

504 the cluster corrected FWE threshold of $P<0.05$ to control for multiple comparisons. The

505 cluster extent for this threshold varied by test and is reported with each analysis.

506 fMRI general linear models 
Three different general linear models (GLMs) were fit to fMRI data:

508 (1) Condition-based GLM

509 The condition-based GLM included separate regressors at the response and feedback

510 phases for the following factors: clustering (recipe, independent), value (positive, negative),

511 presentation number $(1,2,3)$ and accuracy (correct, error). The duration of the boxcar for

512 the response regressors was set to the median of subjects' combined reaction time and slider

513 movement durations. Trial-by-trial deviation from this median duration was included as an

514 additional nuisance regressor as a means of controlling for the influence of RT on a trial-to-

515 trial basis independent of the effects of condition. This GLM also included a nuisance

516 regressor for the time the stimulus display was on screen (i.e. the response window, ISI and

517 feedback).

518 (2) Finite impulse response GLM

519 For ROI-based analyses on contrasts of task conditions, a separate finite impulse

520 response (FIR) GLM was fit to each participant. This model had the identical conditions as

521 the condition-based GLM described immediately above, but used an FIR model with eight

522 time points (16 s window) as a basis function for the hemodynamic response function. This

523 allowed us to contrast activity between conditions within different ROIs without

524 assumptions about the shape of the hemodynamic response function made for simplification

525 in whole-brain and model-based analyses.

526 (3) Model-based value components GLM

527 Finally, we tested for correlates of model-based value terms and their interaction

528 with participants' value ratings (i.e. participant-P(yes)) using a parametric GLM. This model

529 had three boxcar regressors: a response regressor with onsets at stimulus presentation and 
530 durations lasting to the point participants finished moving the slider, a feedback regressor

531 with onsets and durations lasting the presentation of feedback, and a nuisance regressor for

532 the stimulus display. There were eight parametric modulators on the response regressor:

533 participant-P(yes), participant confidence, experienced value, schema value, unfamiliarity,

534 interactions between participant-P(yes) and experienced value, schema value and

535 unfamiliarity, as well as subjective value ratings for ingredients. These interactions served

536 as a filter to focus on neural activity that responded to the influence of these model-based

537 terms on participants' value judgments. We examined the covariance structure of these

538 modulators to assess their independence. The mean $R^{2}$ for all pairs of parametric modulators

539 was 0.08 , while the maximum $R^{2}$ was 0.56 for the correlation between experienced value and

540 participant-P(yes). This stronger correlation was not surprising given that experienced

541 value was a major determinant of participants' P(yes) ratings. The feedback regressor also

542 had two parametric modulators: reward prediction errors (RPE; calculated as the difference

543 between participant-P(yes) and outcomes) and surprise (calculated as the absolute value of

544 RPEs). Parametric modulators were not orthogonalized in this model.

545 Region of interest analyses

546 Regions of interest (ROIs) were defined for testing region-specific hypotheses using

547 unbiased criteria. Specifically, we chose ROIs within a network previously identified by our

548 lab and others as forming a network involved in the storage and retrieval of semantic and

549 episodically encoded information (MTC, aVLPFC, HPC; (Badre, Poldrack, Pare-Blagoev,

550 Insler, \& Wagner, 2005a; Barredo, Verstynen, \& Badre, 2016; Thompson-Schill, D'Esposito,

551 Aguirre, \& Farah, 1997)), and in forming estimates of subjective and expected value (vmPFC,

552 mPFC, striatum; (Barron, Dolan, \& Behrens, 2013; Bartra, McGuire, \& Kable, 2013)). These 
553 ROIs were either defined using masks from the automated anatomical labelling atlas (AAL),

554 or using $10 \mathrm{~mm}$ spheres around peak coordinates from independent studies. These spheres

555 were then masked to only include voxels that were more than $50 \%$ likely to be gray matter

556 in SPM's tissue probability map. The coordinates and references for these ROIs are listed in

557 Table 1.

558 In ROI analyses contrasting conditions, the time course of activity within each ROI

559 was estimated using the MarsBar Toolbox (Brett, Anton, Valabregue, \& Poline, 2002) from

560 the FIR GLM. For each ROI, the peak activity was found for using the grand-average of this

561 timecourse across conditions and subjects (with equal weight for each conditions), and the

562 mean activity \pm 1 bin around this time point was extracted and tested using RM-ANOVAs. In

563 ROI analyses of parametric GLM models, the mean beta weights within ROI voxels were

564 extracted instead.

565 Generalized psychophysical interaction analysis

566 Generalized psychophysical interaction (gPPI) analyses were carried out using the

567 CONN toolbox in conjunction with SPM12 (McLaren, Ries, Xu, \& Johnson, 2012; Whitfield-

568 Gabrieli \& Nieto-Castanon, 2012). The same ROIs described in Table 1 were used as seeds

569 for focused ROI-ROI connectivity analyses and exploratory whole-brain analyses. Task-

570 related activity was regressed out using the condition-based GLM described above, including

571 six regressors for translational and rotational motion and trial-by-trial deviations from the

572 median of reaction time and slider movement durations, as well as additional nuisance

573 regressors including five principal components for both cerebrospinal fluid voxels and white

574 matter, and a regressor for linear trends in the signal. The residuals of this model were then

575 high-pass filtered at $0.008 \mathrm{~Hz}$. 
Following this denoising process, gPPI analyses involved a two-step process. The

577 regressors from the condition-based GLM, the timecourse of each ROI and the interaction of

578 this timecourse with the condition-based regressors were included in a multiple linear

579 regression model. Task-related changes in the relationship between activity in pairs of

580 regions was estimated using the bivariate correlation between these interactions.

581 Correlation coefficients for each subject were Fisher transformed and contrasted between

582 conditions. In a second level analysis, these differences were subjected to a one-sample t-test

583 against zero. Exploratory seed-to-voxel whole brain connectivity analyses were similarly

584 carried out by calculating the bivariate correlation of this interaction term between an ROI

585 and each target voxel. These correlations were then Fisher-transformed and the difference

586 between transformed correlations in task conditions was compared to zero with one-sample

587 t-tests. As with above fMRI analyses, all whole-brain effects were first peak thresholded at $P$

$588<0.001$ and further thresholded at FWE cluster corrected threshold of $P<0.05$ to control for

589 multiple comparisons. Cluster thresholds are reported separately for each analysis.

\section{$590 \quad$ Results}

\section{Behavioral data}

592 To test if participants took advantage of schematic knowledge to make decisions, we

593 first examined whether participants choices reflected customers' recipe preferences on the

594 first presentation of ingredients before seeing any external feedback (Figure 3a). On these

595 trials, participants accepted ingredients associated with the positive value recipe more often

596 than ingredients associated with the negative value recipe and more often than positive

597 value independent ingredients. This was evident in a significant three-way interaction

598 between value, clustering and presentation number on participants' choices (RM-ANOVA: F 
$\left.5992,44=27.20, P<0.0001, \eta_{p}^{2}=0.55\right)$, and direct contrasts between the ingredient types

600 (Bonferroni corrected t-tests: $t(22) \geq 7.79, P^{\prime} s<0.0001, d^{\prime} s \geq 1.62$ ).

601

In contrast, there was no significant difference between acceptance rates for positive

602 and negatively valued independent ingredients on the first presentation. This was as

603 expected, as the ingredients in this condition did not have inferable value based on an

604 association with a positive or negative recipe. The interaction between value and clustering

605 on participants' choices was confirmed with a separate RM-ANOVA on the first presentation

606 only $\left(\mathrm{F}_{1,22}=61.31, P<0.0001, \eta_{p}^{2}=0.73\right)$. Participants accepted ingredients associated with

607 the negative value recipe numerically less often than negative independent ingredients, but

608 this difference was not significant after correction for multiple comparisons. Overall, the

609 choice results indicate that participants successfully inferred value information from recipe

610 associations, though these effects were stronger for the positive recipe.

611 There was clear evidence that participants learned ingredient values from feedback.

612 Participants made choices consistent with the values of ingredients more often in the second

613 and third presentations than in the first in all conditions, demonstrating retention of

614 feedback from previous trials (Bonferroni corrected t-tests: $t$ 's $(22) \geq 4.54, P^{\prime} s<0.01, d^{\prime} s \geq$

615 0.95). Similarly, participants' rate of value consistent choices increased significantly from the

616 second presentation to the third in all conditions, except for independent positive

617 ingredients (Bonferroni corrected t-tests: $t^{\prime} s(22) \geq 4.69, P^{\prime} s<0.01, d^{\prime} s \geq 0.98$ ).

618 Notably, participants also tended to reject both positive and negative independent

619 ingredients in the first presentation significantly more often than chance (One sample t-

620 tests: $\left.t^{\prime} s(22) \geq 8.69, P^{\prime} s<0.0001, d^{\prime} s \geq 1.81\right)$. Thus, participants strategically avoided

621 endorsing independent ingredients before learning their values through feedback. 
To test how participants' belief in their choices changed during the task, we next

623 examined how reported confidence changed on correct responses (Figure 3b). Confidence

624 increased with repeated presentations, and was also greater overall for recipe and positive

625 value ingredients (RM-ANOVA: F's $\geq 27.88, P \leq 0.0001, \eta_{p}^{2} \geq 0.58$ ). Confidence for acceptance

626 of positive recipe ingredients was significantly higher than positive independent ingredients

627 on the first presentation (Bonferroni corrected t-test: $t(20)=4.57, \mathrm{P}<0.01, d=0.99$ ) and

628 third presentation (Bonferroni corrected t-test: $t(20)=4.96, \mathrm{P}<0.01, d=1.08$ ). However,

629 there was no comparable difference in confidence for rejections of negative recipe and

630 independent ingredients, resulting in a significant interaction between clustering and value

631 (RM-ANOVA: $\left.\mathrm{F}_{1,20}=9.28, P=0.006, \eta_{p}^{2}=0.32\right)$,

Confidence also increased significantly from the first presentation to the second and

633 third presentations in all conditions (Bonferroni corrected t-tests: $t^{\prime} s(20) \geq 4.57$, P's $\leq 0.01$,

$634 d$ 's $\geq 1.29$ ), and from the second to the third presentation in all conditions, except for negative

635 recipe ingredients (Bonferroni corrected t-tests: $t$ 's $(20) \geq 3.98, \mathrm{P}^{\prime} \mathrm{s} \leq 0.05, d^{\prime} \mathrm{s} \geq 0.87$ ), leading

636 to a modest interaction of value and presentation number $\left(\mathrm{F}_{2,40}=3.34, P=0.04, \eta_{p}^{2}=0.14\right)$.

637 Similar to reported confidence, participants' reaction times were also strongly

638 influenced by experience with ingredients with stronger decrements in subsequent

639 presentations of positive recipe ingredients (Figure 3c). There was a significant interaction

640 between value with presentation number $\left(\mathrm{F}_{2,40}=8.50, P=0.0008, \eta_{p}^{2}=0.30\right)$, with larger

641 reductions in reaction times for positive ingredients, consistent with better recall. There was

642 also an interaction between value and clustering (RM-ANOVA: $\mathrm{F}_{1,20}=4.66, P=0.04, \eta_{p}^{2}=$

643 0.19), owing to comparatively bigger effect of value on recipe ingredients compared to 
644 independent ingredients. Responses in the first presentation did not significantly differ by 645 cluster condition or value.

646 In anticipation of analyzing the effects of memory retrieval failures on brain activity,

647 we also tested if errors were associated with lower confidence and longer reaction times, as

648 would be expected for retrieval failures. We collapsed across value levels and the second and

649 third presentations to have enough error trials per participant for this analysis. Confidence

650 ratings were influenced by significant interactions between accuracy and clustering (Figure

651 3d; RM-ANOVA: $\mathrm{F}_{1,21}=15.43, P=0.0007, \eta_{p}^{2}=0.42$ ), and accuracy and presentation number

$652\left(\mathrm{~F}_{1,21}=43.69, P<0.0001, \eta_{p}^{2}=0.67\right)$. On the first presentation, participants were

653 significantly more confident on correct responses for recipe ingredients than independent

654 ingredients (Bonferroni corrected t-tests: $t(21)=3.77, \mathrm{P}<0.05, d=0.80$ ), and error

655 responses on recipe ingredients (Bonferroni corrected t-tests: $t(21)=5.60, \mathrm{P}<0.01, d=$

656 1.19). There was no comparable significant difference in confidence for correct and error

657 responses for independent ingredients in the first presentation. We confirmed that the

658 effects of accuracy differed between clustering conditions on this first presentation by

659 separately testing this interaction (RM-ANOVA: $\mathrm{F}_{1,21}=14.34, P=0.001, \eta_{p}^{2}=0.40$ ). Thus,

660 confidence on the first presentation appeared to selectively depend on retrieval of relevant

661 recipe associations. Confidence was also higher on accurate responses compared to errors

662 on the second and third presentations of both recipe and independent ingredients

663 (Bonferroni corrected t-tests: $t$ 's $(21) \geq 6.64$, $\mathrm{P}^{\prime} \mathrm{s}<0.0001, d=1.42$ ).

664 Reaction times were influenced by an interaction of accuracy and presentation 665 number (Figure 3e; RM-ANOVA: $\mathrm{F}_{1,21}=49.84, P<0.0001, \eta_{p}^{2}=0.70$ ), but there was only a 666 trend toward an interaction between accuracy and clustering $\left(\mathrm{F}_{1,21}=3.81, P=0.06, \eta_{p}^{2}=\right.$ 
667 0.15), and no three-way interaction. Participants were significantly faster on correct

668 responses than errors on the second and third presentations of both recipe and independent

669 ingredients (Bonferroni corrected t-tests: $t$ 's $(21) \geq 5.03$, $\mathrm{P}^{\prime} \mathrm{s}<0.01, d \geq 1.07$ ). This pattern is

670 consistent with longer reaction times on errors reflecting retrieval failures (Ratcliff, 1978).

671 In contrast, there were no significant differences in reaction times for correct and error

672 responses in the first presentation for either clustering condition.

673 In summary, behavioral results established that participants were able to infer the

674 value of ingredients based on their recipe association and used these inferred values to make

675 choices. Participants also showed a generally risk averse attitude on this first presentation

676 by tending to reject independent ingredients. On subsequent presentations of an ingredient,

677 participants accurately retrieved explicit memory of deterministic feedback to make faster,

678 more confident decisions. This was the case across both recipe and independent items.

679 However, participants continued to benefit from recipe associations in these later

680 presentations, with generally faster reaction times and greater confidence for correct

681 responses in this condition compared to independent ingredients. Finally, participants

682 tended to asymmetrically utilize knowledge about the positive recipe more than the negative

683 recipe throughout all trials.

684 Main effects of task conditions in whole-brain analyses

685 We hypothesized that vmPFC and striatum would respond to schema-based and 686 experienced values of ingredients respectively, and interact with different memory systems

687 in retrieving and constructing these values. Thus, we predicted that vmPFC activity would

688 be modulated by schema values more than directly experienced values, and the opposite

689 would be true of the striatum. We also predicted that a network of regions, including the 
690 striatum, HPC and middle temporal cortex would be engaged by successful retrieval (Spaniol

691 et al., 2009), while aVLPFC would be engaged by retrieval difficulty (Badre, Poldrack, Pare-

692 Blagoev, Insler, \& Wagner, 2005b). We first tested how ingredient clustering, value,

693 presentation number and accuracy affected activity using simple contrasts from our

694 condition-based GLM at a whole-brain level (Table 2). These analyses were filtered to focus

695 on correct responses, excluding trials where participants may have made errors due to

696 failure to appropriately recall information relevant to ingredient values, except where trials

697 with different response accuracy were directly contrasted.

698 Recipe ingredients elicited greater activity than independent ingredients in medial

699 OFC (Figure 4a), but not elsewhere. Ingredients associated with positive value elicited

700 greater activity in OFC and medial PFC, as well as the ventral striatum (Figure 4b). Compared

701 with later ingredient presentations, the first encounter with an ingredient was associated

702 with widespread activity in lateral PFC, including left VLPFC, inclusive of the aVLPFC, and

703 the caudate nucleus (Figure 4c). Activity in left MTC, bilateral HPC and putamen was higher

704 in correct responses compared to errors across all presentations (Figure 4d).

705 Region of interest analyses

706 To follow-up the whole-brain voxel-wise contrasts, we tested for more specific task-

707 dependent changes in activity within a set of a priori regions of interest (ROIs) defined in

708 Table 1 using a finite impulse response (FIR) model of the hemodynamic response. We were

709 primarily interested in how the clustering status of ingredients influenced value-related

710 activity across ingredient repetitions on correct responses. Here we focused on vmPFC and

711 the striatum, since we had a priori expectations that these regions would differentially

712 process value related to episodic and schematic memory. As the medial frontal wall is 
713 functionally heterogeneous (Braga \& Buckner, 2017; Roy, Shohamy, \& Wager, 2012), we

714 included two ROIs from this region, one that was defined by a meta-analysis of studies of

715 expected-value (Bartra et al., 2013), and a more dorsal ROI (mPFC) that responded to shared

716 associations between components of a recipe schema in another experiment and was

717 independent and non-overlapping with the vmPFC ROI (Barron et al., 2013). This dorsal

718 mPFC ROI responded to ingredient value, but not any other task manipulations.

719 vmPFC was more active for positive than negative expected value, evident in a main

720 effect of value (Figure 5a; RM-ANOVA: $\mathrm{F}_{1,20}=9.13, P=0.007, \eta_{p}^{2}=0.31$ ), consistent with the

721 whole-brain analysis. Activity in this region was greater for positive compared to negative

722 recipe ingredients in the third presentation (Bonferroni corrected t-test: $t(20)=4.90, \mathrm{P}<$

$7230.01, d=1.07$ ), but there was no comparable value difference for independent ingredients

724 (Bonferroni corrected t-test: $t(20)=1.13, \mathrm{P}>0.05, d=0.25)$. There was no significant

725 difference between vmPFC activity in response to positive and negative recipe ingredients

726 on the first presentation, leading to a three-way interaction between value, presentation

727 number and clustering $\left(\mathrm{F}_{1,20}=5.06, P=0.01, \eta_{p}^{2}=0.20\right)$. This null result of value on the first

728 presentation was not merely due to the ROI selection; an exploratory whole-brain contrast

729 of positive and negative recipe conditions on the first presentation in the condition-based

730 GLM was similarly null. vmPFC activity was also generally higher in later presentations,

731 reflected in a marginal main effect of presentation number $\left(\mathrm{F}_{2,20}=3.29, P=0.05, \eta_{p}^{2}=0.14\right)$,

732 but there was no significant main effect of clustering $\left(\mathrm{F}_{1,20}=1.42, P=0.2, \eta_{p}^{2}=0.06\right)$.

733 Within the striatum ROI, there was greater activation for positive than negative value,

734 evident in a strong main effect of value (Figure $5 \mathrm{~b} ; \mathrm{F}_{1,20}=9.17, P=0.007, \eta_{p}^{2}=0.31$ ), 
735 consistent with the observation from the whole brain voxel-wise analysis. However, there

736 were no other significant main effects or interactions.

737 We hypothesized that value judgments in this task would rely on different neural

738 systems supporting different types of memory. In particular, we predicted that retrieval of

739 values associated with independent ingredients learned through feedback would place

740 greater demands on the HPC, while the MTC would be involved more in trials where

741 semantic memory about recipes was needed to infer ingredient values and aVLPFC would be

742 engaged by greater retrieval demands on the first presentation. To test these predictions, we

743 evaluated the effects of clustering status, presentation number and accuracy in ROI-based

744 analyses of the HPC, MTC and aVLPFC. We collapsed across value conditions and combined

745 presentations two and three into a single level to have enough error trials for this analysis.

746

HPC activity was greater on accurate trials, confirming the whole-brain results

747 (Figure 5c; RM-ANOVA: $\mathrm{F}_{1,21}=13.14, P=0.002, \eta_{p}^{2}=0.38$ ). HPC activity was also generally

748 lower on later presentations $\left(\mathrm{F}_{1,21}=6.54, P=0.02, \eta_{p}^{2}=0.24\right)$. The effect of accuracy on HPC

749 activity was modulated marginally by clustering $\left(\mathrm{F}_{1,21}=4.65, P=0.04, \eta_{p}^{2}=0.18\right)$.

750 Surprisingly, we found greater HPC activity on correct trials than error trials for recipe

751 ingredients, collapsing across presentations (Bonferroni corrected t-test: $t(21)=3.47, \mathrm{P}<$

$7520.01, d=0.74$ ), but no such accuracy effect for independent ingredients. There was no main

753 effect of clustering, or three-way interaction of clustering, accuracy and presentation.

754 MTC activity responded to an interaction of clustering and presentation number

755 (Figure 5d; $\mathrm{F}_{1,21}=5.60, P=0.03, \eta_{p}^{2}=0.21$ ). Posthoc tests collapsed across accuracy revealed

756 a significantly greater reduction in MTC activity for independent ingredients in the second

757 and third presentations compared to the first presentation (Bonferroni corrected t-test: 
$758 t(21)=2.87, \mathrm{P}<0.05, d=0.61)$, but no significant difference for recipe ingredients. While

759 MTC seemed to be engaged on the first presentation in all conditions, this reduced in

760 subsequent presentations for independent ingredients, consistent with less reliance on

761 semantic knowledge in this condition.

762 aVLPFC activity was greater on the first presentation compared to the second and

763 third, consistent with the whole-brain analysis (Figure 5e; Presentation Main Effect: $\mathrm{F}_{1,21}=$

$76410.76, P=0.004, \eta_{p}^{2}=0.34$ ). The response of aVLPFC did not change with accuracy or

765 clustering status, or with any interaction between task variables.

766 In summary, ROI-based analyses confirmed whole-brain contrasts demonstrating

767 that the vmPFC and striatum carried information about ingredient values. Unlike the

768 striatum, value-related activity in vmPFC was greater for recipe ingredients compared to

769 independent ingredients in later presentations, but not in the first presentation where the

770 demand for inferring ingredient values from recipe associations was the greatest. In line with

771 our predictions, the MTC responded to differential demands on semantic memory, though

772 this was only true in later presentations. aVLPFC activity diminished with presentation

773 overall, perhaps in line with greater retrieval demands early on. While the HPC responded

774 differentially to retrieval success and failures in later presentations, this effect was

775 surprisingly greater for recipe ingredients than independent ingredients, possibly due to the

776 stronger encoding of these recipe ingredients, evident in participants' behavior on these

777 trials.

778 Computational model comparison

779 We had hypothesized that vmPFC would be preferentially engaged by schema-based

780 values, while the striatum would be involved in evaluating the contents of episodically 
781 encoded value information. While value-related differences in vmPFC activity grew larger in

782 later presentations of recipe ingredients compared to independent ingredients, this change

783 in activity could not be easily differentiated from value information learned from feedback.

784 Importantly, this condition-based analysis also ignores the continuous nature of associations

785 between independent ingredients and recipes. For example, strawberries are considered

786 part of the 'cake' cluster, but also have some association with 'pie.' Further, filtering trials by

787 accuracy based on participant responses and task-defined values ignores that responses to

788 independent ingredients on the first presentation are made without any useful experienced

789 or schematic value information, and ignores systematic biases or strategies participants may

790 use in making choices that are not explicitly captured by our experimental design (e.g. a bias

791 against endorsing independent ingredients on their first presentation).

792 To better separate these components of expected value for testing our first two

793 hypotheses regarding the neural systems supporting valuation of information from different

794 memory sources, we developed a computational model of behavior to estimate the

795 contribution of information learned directly from experience and inferred from schematic

796 associations to participants' choices in each trial. We then used these values in a model-based

797 parametric GLM to determine the correlates of this information during value judgment.

799 in this task that are described in more detail in the Materials and Methods section. Briefly,

800 each ingredient had an 'experienced value' (EV), which depended on feedback and decayed

801 over time, and a 'schema value' (SV), which was derived from the differences in associative

802 strength between an ingredient and the positive and negative recipes. The associative

803 strength of an ingredient with each recipe was based on a latent semantic analysis of 
804 normative ingredient-recipe associations (Dumais, 2004; Landauer et al., 1998). Each of

805 these value terms were weighted by a temperature parameter that determined their

806 contribution to the model's output, a continuous estimate of the probability that it would

807 endorse an ingredient, which we will refer to as model-P(yes).

808 Starting with these assumptions, we followed a step-wise process of adding

809 parameters to a model with these features and using cross-validation to compare the new

810 model against the best simpler model. This allowed us to test if the new model better

811 explained participants' behavior on trials that were held out from the dataset used to fit

812 parameter values. Figure 6 shows the difference in the negative log likelihoods for these

813 alternative models against the base model. Please see the methods section for a fuller

814 accounting of the parameters in the best fitting model and alternative models in this figure.

815 The final best fitting model allowed for a more sophisticated parameterization of SV

816 where the number of dimensions in the latent semantic analysis of recipe-ingredient

817 associations was allowed to vary. This parameter essentially controlled the depth of recipe

818 information considered in computing SV (from simple distinctions like savory-sweet to more

819 fine-grained information about particular recipes; Figure 2). The model also discounted the

820 influence of $S V$ based on the memory strength of the ingredients' experienced value, with

821 schema values contributing less to ingredient values when these experienced values were

822 stronger.

824 or passing in the best fitting model. Unfamiliarity was operationalized as the opposite of the

825 unsigned experienced value of items. In other words, unfamiliarity was highest when

826 ingredients were seen on the first presentation, or when ingredients had not been seen for 
827 many trials and memory for their experienced value had decayed. This parameter thus

828 effectively accounted for variance in participants' willingness to bet on ingredients when

829 information about their experienced values was weaker. Lastly, an additional free parameter

830 acted as an intercept term in the decision function, which effectively acted as a fixed bias on

831 the model's tendency to bet on ingredients.

832 Computational model validation

833 To ensure that our computational model was capturing participants' decisions and

834 understand how it was accomplishing this, we carried out a series of analyses to validate its

835 parameters and behavior. We first examined if there were general tendencies for the two

836 bias and schema similarity temperature parameters towards positive or negative values by

837 conducting two-tailed, one-sample t-tests against zero (Table 3). While the unfamiliarity bias

838 parameter was significantly lower than zero $(t(22)=4.13, P=0.0004, d=0.86)$, the opposite

839 was true of the fixed bias parameter $(t(22)=4.69, P=0.0001, d=0.98)$.

840 It is possible that this unfamiliarity term simply captures a bias to pass on the first

841 presentation of ingredients rather than an effect of unfamiliarity more generally - i.e. the

842 additional effect of the passage of time and repeated presentations. To examine if there was

843 an effect of unfamiliarity on participants' willingness to endorse an ingredient in later trials,

844 we used a logistic regression in each participant to test if there was a relationship between

845 the model-based unfamiliarity term on betting decisions on the second and third

846 presentations. A one-sample t-test revealed that these logistic regression coefficients were

847 significantly lower than zero across participants, indicating a persistent negative influence

848 for unfamiliarity on participants' decisions in later presentations $(t(22)=3.62, \mathrm{P}=0.001, d$ 
$849=0.75)$. Thus, this unfamiliarity term captures additional variance in decision behavior that

850 beyond an aversion to betting on the first presentation of an ingredient.

851 We also examined the covariance between parameters recovered from fitting this

852 model to participants' behavioral data to test their independence (Table 3). The fixed bias

853 parameter had a strong negative correlation with the unfamiliarity bias parameter,

854 indicating that this fixed bias in effect acted to counter the aversion to betting related to

855 unfamiliarity. Despite this strong relationship, both parameters resulted in large

856 improvements in model fitness when included together (Figure 6), indicating that they were

857 complimentary.

858 To confirm that all of these parameters could be uniquely recovered in our model

859 fitting procedure, we generated 100 simulated participants with randomly assigned

860 parameters using the best fitting model. We reliably and accurately recovered all parameters

861 in these data, with high correlations between simulated and recovered parameters (Pearson

$\left.862 r^{\prime} \mathrm{s}(98) \geq 0.82, P^{\prime} \mathrm{s}<0.0001\right)$. However, as these data were simulated from randomly

863 generated parameters, they did not share the covariance structure of the parameter

864 estimates fit to participants' behavior described above. To assure that we could recover

865 these parameters with the same covariance, we fit the model to simulated data generated

866 using parameter estimates from the model fit to participants' behavior. For all seven

867 parameters, there was a near perfect correlation between the parameter estimates from

868 participants' behavior and the simulated data (Pearson $r^{\prime} s(21) \geq 0.99, P^{\prime} s<0.0001$ ).

869 Finally, we generated simulated behavior from the model using participants'

870 recovered parameters to test that the model recapitulated key features of participants'

871 behavior across task conditions. To directly compare the model and participants' behavior, 
872 we focused on participants' continuous estimate of whether or not they should endorse an

873 ingredient, based on position of the slider between the 'yes' and 'no' ends of the bar (i.e.

874 participant-P(yes)). We compared these ratings to the model-P(yes) measure, and

875 transformed model-P(yes) to confidence based on the distance from the point of maximum

876 uncertainty (i.e. 0.5). Participant-P(yes) ratings followed a similar pattern to their averaged

877 binary choices, including key features on the first presentation like higher P(yes) for positive

878 recipe ingredients compared to negative recipe, and positive independent, ingredients, and

879 a bias against endorsing independent ingredients (data not shown)

880 We analyzed model-P(yes) and confidence ratings in the same way as participants'

881 responses. Like with participants' choices, there was a strong three-way interaction between

882 value, clustering and presentation number (Figure 7a; RM-ANOVA: F 2,44 $=73.09, P<0.0001$,

$\left.883 \eta_{p}^{2}=0.77\right)$. The model used recipe information to make decisions on the first presentation,

884 with higher model-P(yes) ratings for positive recipe ingredients over negative recipe

885 ingredients, and over independent positive ingredients (Bonferroni corrected t-tests: $t$ 's(22)

$886 \geq 9.12, \mathrm{P}^{\prime} \mathrm{s}<0.0001, d^{\prime} \mathrm{s} \geq 1.90$ ). There was also no significant difference in model-P(yes) for

887 positive and negative independent ingredients on this first presentation. Unlike real

888 participants, the model gave significantly lower ratings to negative recipe ingredients than

889 negative independent ingredients on the first presentation (Bonferroni corrected t-test:

$890 t(22)=9.52, \mathrm{P}<0.0001, d=1.99)$, suggesting that it used information about the negative

891 recipe more than participants did. Model-P(yes) on the first presentation of independent

892 ingredients trended negatively, but was not significantly lower than chance $(t(22)=1.65, \mathrm{P}$

$893=0.1, d=0.34$ ), indicating a modest difference from participant behavior. In subsequent

894 presentations, the model also followed the values of options learned through feedback, 
895 hewing closer to ingredient values in presentation two and three compared to one

896 (Bonferroni corrected t-tests: $t$ 's(22) $\geq 8.19$, $\mathrm{P}^{\prime} \mathrm{s}<0.0001, d \geq 1.71$ ), and presentation three

897 compared to two (Bonferroni corrected t-tests: $t$ 's(22) $\geq 6.03$, $\mathrm{P}^{\prime} \mathrm{s}<0.01, d \geq 1.26$ ).

898 Similarly, we examined whether the model's predicted confidence followed the same

899 pattern as participants' responses across task conditions on trials where participants

900 responded correctly (Figure 7b). Model confidence rose predictably with presentation

901 number (RM-ANOVA: $\left.\mathrm{F}_{2,40}=165.64, P<0.0001, \eta_{p}^{2}=0.89\right)$, and was higher overall for recipe

902 ingredients than independent ingredients ( $\left.\mathrm{F}_{1,20}=46.48, P<0.0001, \eta_{p}^{2}=0.70\right)$, however

903 there was no main effect of value $\left(\mathrm{F}_{1,20}=1.96, P=0.2, \eta_{p}^{2}=0.09\right)$. Model confidence was also

904 notably higher for both positive and negative recipe ingredients compared to independent

905 ingredients on the first presentation (Bonferroni corrected t-tests: $t^{\prime} s(20) \geq 4.50$, P's $<0.01$,

$906 d$ 's $\geq 0.98)$, rather than just positive recipe ingredients.

907 In summary, our model captured most core aspects of participants' behavior in this

908 task, including utilization of recipe-based values. The model was also able to recreate the

909 basic pattern of participants' reported confidence rising with repeated presentations, with

910 benefits for positive value and recipe associations. However, the model gave greater weight

911 to the negative value recipe than participants and underestimated the effects of value on

912 confidence. While an alternative model with asymmetric weights for positive and negative

913 recipes improved model fitness, this addition did not survive our model selection process

914 (Figure 6), possibly due to variance between participants in the extent of this asymmetry.

915 The model also underestimated the effects of unfamiliarity on P(yes) on the first

916 presentation of independent ingredients, though was trending in the same direction as

917 participants. 
Model-based fMRI

We had hypothesized that vmPFC would be engaged more by values inferred from

920 schematic associations than directly experienced values, which we hypothesized would be

921 tracked by activity in the striatum. Our computational model showed that ingredient values

922 could be captured by a combination of experienced value, schema value and unfamiliarity,

923 and allowed us to leverage these terms in testing these hypotheses by examining their

924 correlates in brain activity.

925 To focus on brain activity that responded to the influence of these model-based value

926 terms on participants' value judgments, we included interactions between these three terms

927 and participant-P(yes) in our GLM. These interactions selectively captured activity that

928 responded to the conjunction (or agreement) between the participants' responses with

929 experienced and schema values (e.g. when schema value was high and participant-P(yes)

930 were high, or when both were low). The interaction of unfamiliarity with value ratings

931 similarly let us capture where activity increased in response to higher participant-P(yes)

932 when unfamiliarity was high, or lower participant-P(yes) when unfamiliarity was low. In

933 doing so, we could focus on activity where behavior and model predictions were in

934 agreement. In contrast, the main effects of these model-based terms only captured the extent

935 to which correlates of this information were present in the brain, independent of

936 participants' response. This analysis may seem circular, as we are looking for correlates of

937 an interaction between these model-based terms with the behavior to which these terms

938 were fit. However, the fMRI correlates that are the dependent measure in this analysis were

939 not part of the model fitting, breaking any potential circularity in our results. This GLM also

940 included parametric modulators for participants' confidence ratings and participants' 
941 subjective value ratings (provided separately in a different task) on the response regressor

942 as nuisance terms.

943 Whole-brain cluster corrected analyses found no significant main effects of

944 participant-P(yes), experienced or schema-based value, or participants' subjective value

945 ratings. There were significant correlates for the main effect of unfamiliarity, with increased

946 activity in lateral PFC, visual cortex and several other regions, similar to the main effect of

947 presentation number in the condition-based contrast above. There were also significant

948 main effects for response confidence in the superior frontal gyrus and visual cortex (Table

$9494)$.

951 in the left and right middle temporal gyrus, right VLPFC and bilateral supramarginal gyri

952 (Figure 8a). The interaction of experienced value and participant P(yes) was also associated

953 with activity in medial OFC and a large area of the medial superior frontal gyrus and left

954 angular gyrus (Figure 8b). There were no clusters that passed the whole-brain FWE cluster-

955 corrected threshold for the interaction of unfamiliarity and participant-P(yes). However,

956 given the challenge of detecting cluster corrected effects in deep brain structures, we applied

957 a small volume correction using the striatum ROI. This analysis found that a cluster in the

958 bilateral caudate nucleus responded to the interaction of unfamiliarity with participant-

959 P(yes) (Figure 8c; FWE-corrected $P<0.05$, MNI: 18, 20, -4). Whole brain peak activations for

960 this GLM are described in Table 4.

961 We next specifically compared how activity related to participant-P(yes) in vmPFC

962 and striatum, the two regions we identified a priori as being associated with valuation, was

963 modulated by experienced value, schema value and unfamiliarity. We extracted the mean 
964 beta coefficients for the interaction between participant-P(yes) and these three terms in the

965 vmPFC and striatum ROIs. The interaction of participant-P(yes) with experienced and

966 schema value was significant in vmPFC (one-tailed, one sample t-tests against zero: $t(22)=$

$9672.38, P \leq 0.01, d$ 's $\geq 0.50$ ). There was also a significant effect for the interaction of schema

968 value with participant-P(yes) in the striatum $(t(22)=2.05, P=0.03, d=0.43)$, but not for

969 the interaction of participant-P(yes) with experienced value. Further, the interaction of

970 participant-P(yes) with unfamiliarity was significant in the striatum $(t(22)=2.30, P=0.02$,

$971 d=0.48$ ), but not vmPFC. Thus, vmPFC responded to greater agreement between both

972 experienced and schema values with participant-P(yes), while striatum activity increased in

973 response to participant-P(yes) when unfamiliarity was high, as well as the agreement of

974 schema values with participant-P(yes).

975 To examine whether ROIs differed in their response to these value-related terms, we

976 then tested an ROI (striatum, vmPFC) by modulator (interactions of participant-P(yes) with

977 experienced value, schema value and unfamiliarity) interaction on these average beta

978 coefficients (Figure 9). This interaction was highly significant $\left(\mathrm{F}_{2,44}=8.67, P=0.007, \eta_{p}^{2}=\right.$

$9790.28)$, as was a main effect of ROI ( $\left.\mathrm{F}_{1,22}=10.59, P=0.004, \eta_{p}^{2}=0.32\right)$, but not modulator ( $\mathrm{F}$

$9802,44=0.10, P=09, \eta_{p}^{2}=0.005$ ). To determine the source of this interaction, we compared the

981 difference of the betas in the VMPFC and striatum for each modulator (i.e. the effect of each

982 interaction between ROIs). This difference was significantly greater for the interaction of

983 participant-P(yes) with experienced value compared to both schema value and unfamiliarity

984 (Bonferroni corrected t-tests $t^{\prime} \mathrm{s}(22) \geq 3.09, P^{\prime} \mathrm{s}<0.05, d \geq 0.64$ ), but there was no comparable

985 significant difference between schema value and unfamiliarity. 
To summarize, vmPFC activity reflected the influence of experienced and schema

987 values on participants' value judgments, and preferentially reflected the influence of

988 explicitly experienced values on participants' value judgments compared to the striatum.

989 Striatal activity reflected the effect of unfamiliarity on participants' value judgments and

990 values inferred from a schema, but not value information stored directly in memory.

991 Generalized psychophysical interactions

992 We hypothesized that differential demand on memory systems during decision-

993 making would also lead to distinct patterns of functional connectivity, reflecting the

994 contributions of different memory stores to the assessment of options. vmPFC has reciprocal

995 connections with regions of middle temporal cortex (MTC) and anterior ventrolateral

996 prefrontal cortex (aVLPFC) (Price, 2007; Saleem, Kondo, \& Price, 2008). Both vmPFC and

997 striatum also receive input from the hippocampus (HPC). We were principally interested in

998 whether vmPFC and MTC connectivity increased with greater demands on schema-based

999 decision-making, and whether striatum and HPC connectivity increased during the

1000 assessment of directly experienced values. To address this hypothesis, we carried out

1001 generalized psychophysical interaction (gPPI) analyses that contrasted how connectivity

1002 between pairs of ROIs listed in Table 1 changed between task conditions, as well as more

1003 exploratory seed-to-voxel whole-brain gPPI analyses focused on vmPFC, MTC, HPC and

1004 striatum.

1005 We first examined whether connectivity with VMPFC and MTC was modulated by the

1006 schema-based value of ingredients on correct responses in the first presentation - i.e. when

1007 these values are correctly inferred from schematic knowledge and not retrieved from

1008 experience. Within an MTC seed, there was increased connectivity with vmPFC on the first 
1009 presentation of positive recipe ingredients compared to negative recipe ingredients

1010 (Bonferroni corrected t-test: $t(21)=4.00, P<0.01, d=0.85)$, but not any other ROI $(P \geq 0.2$,

1011 Bonferroni corrected). Similarly, using vmPFC as a seed ROI revealed increased connectivity

1012 with the MTC ROI in the same contrast (Bonferroni corrected t-test: $t(21)=3.68, P<0.05, d$

$1013=0.78$ ), but not any other ROI. An exploratory whole-brain seed-to-voxel analysis on this

1014 contrast with the MTC ROI as a seed found that this region had significantly greater

1015 connectivity with a cluster on the medial frontal wall overlapping with our vmPFC ROI

1016 (Figure 10a, MNI: -2, 46, -12), and a second cluster in lateral occipital cortex (MNI: -52, -68,

1017 12). The same whole-brain ROI-to-voxel contrast with the vmPFC ROI as a seed did not reveal

1018 any significant clusters at an FWE cluster-corrected threshold.

1019 These data provide evidence that VmPFC and MTC connectivity changed depending

1020 on the value of recipe ingredients on the first presentation. To examine whether this effect

1021 might depend on successful retrieval of relevant schema-value information, we tested the

1022 interaction of value and accuracy on the first presentation of recipe ingredients. This

1023 interaction was significant when either vmPFC and MTC was used as a seed (Bonferroni

1024 corrected t-tests: $\left.t^{\prime} \mathrm{s}(20) \geq 2.95, P<0.05, d \geq 0.64\right)$, but not between these seeds and any

1025 other ROIs. There was no main effect of accuracy on connectivity between ROIs on these

1026 trials. We further examined whether this value related increase in vmPFC-MTC connectivity

1027 was higher on the first presentation than subsequent presentations by testing the interaction

1028 of presentation number and value for recipe ingredients, but this interaction did not reach

1029 significance after correction for multiple comparisons in either seed.

1030 Thus, vmPFC-MTC connectivity increased in response to retrieval of positive values

1031 inferred from schematic knowledge on this first presentation, and though this increase was 
1032 greater on correct trials compared to errors, there were no main effects of clustering

1033 condition or accuracy. These null effects could partially stem from participants attempting

1034 to retrieve schematic associations on the first presentation in all conditions, consistent with

1035 greater MTC activity in these presentations compared to repetitions in the independent

1036 condition.

1037 We also tested whether connectivity between MTC and vmPFC and any other ROIs

1038 depended on clustering status by contrasting the recipe and independent conditions on the

1039 first presentation, collapsing across value. There was no significant change in connectivity

1040 between MTC, vmPFC and any other ROIs in this contrast. Exploratory seed-to-voxel

1041 analyses for both of these contrasts were similarly null.

1042 Next, we investigated if connectivity between with the HPC and striatum and other

1043 ROIs depended on the value of options in the second and third presentations. Contrasting

1044 positive and negative independent ingredients in these trials, we did not find any significant

1045 increase in connectivity between HPC and striatum with each other or any other region in

1046 ROI-to-ROI, or seed-to-voxel analyses. We further examined if connectivity with these ROIs

1047 was higher for positive than negative ingredients in the independent condition relative to

1048 the recipe condition by testing the interaction of value and clustering. This analysis was

1049 similarly null for both seeds.

1050 We also tested if HPC and striatum connectivity depended on demands for retrieving

1051 previously experienced feedback associated with particular ingredient instance. Collapsing

1052 across value conditions, we contrasted independent and recipe ingredients on the second

1053 and third presentations where ingredient values could be retrieved from episodic memory,

1054 with the expectation that demand on episodic memory would be greater in the independent 
1055 condition. There was no significant difference in connectivity between the striatum or HPC

1056 with any other ROIs in this contrast. However, a seed-to-voxel whole-brain analysis with the

1057 HPC seed showed significantly greater connectivity with a cluster in the right putamen and

1058 insula (Figure 10b; MNI: 26, 10, 0). The reverse seed-to-voxel analysis with the striatum ROI

1059 as a seed did not reveal significant clusters within a small-volume corrected HPC ROI.

1060 To test if this increase in HPC and hippocampus connectivity was related to retrieval

1061 success we also contrasted correct and error responses on the second and third presentation

1062 of independent ingredients. Using the HPC as a seed in a seed-to-voxel analysis, we found

1063 increased connectivity with this ROI and the right putamen (MNI: 28, 2, 4) after small volume

1064 correction within the striatum ROI. An exploratory whole-brain analysis also found that HPC

1065 significantly increased connectivity with clusters in bilateral anterior rostrolateral PFC and

1066 right insula (Figure 10c). However, there were no significant differences in connectivity

1067 between these conditions in ROI-to-ROI contrasts with either HPC or striatum as seeds. The

1068 reverse ROI-to-voxel analysis with the striatum ROI as a seed did not reveal significant

1069 increase in connectivity in a small-volume corrected HPC ROI. All peaks from seed-to-voxel

1070 analyses are reported in Table 5.

1071 In summary, we found that vmPFC-MTC connectivity depended on recipe value

1072 associations on the first presentation where this information was key to guiding choice.

1073 Meanwhile, HPC increased connectivity with the right putamen in response to greater

1074 demand on episodic memory compared to trials where schema memory could still be used,

1075 and trials where value information was not successfully retrieved.

\section{Discussion}


1078 distinct memory sources. We had put forward three main hypotheses: (1) vmPFC is

1079 preferentially involved in inferred value judgments based on schematic world knowledge,

1080 (2) striatum is preferentially involved in representing experienced values, (3) vmPFC and

1081 striatum interact with distinct memory networks in line with their respective involvement

1082 in building values from schematic knowledge and experience.

$1083 \quad$ Our results were inconsistent with these a priori hypotheses, but yielded new insights

1084 into the brain systems linking memory with value-based decision-making. Striatum

1085 responded to the effect of the unfamiliarity of options on participants' value judgments and

1086 schema-based values. In contrast, vmPFC responded to the influence of value judgments

1087 based both on direct experience and schema memory. Analysis of connectivity between

1088 these regions and memory stores revealed greater connectivity between the striatum and

1089 HPC in response to demands on recall of experienced values, while vmPFC and MTC

1090 connectivity increased in response to values derived from schema memory. Thus, vmPFC

1091 represented retrieved value information from multiple memory sources, and coordinated

1092 with MTC when value was inferred. In contrast, the striatum incorporated basic memory

1093 strength into the decision process, and increased connectivity with HPC in response to

1094 demand for retrieval of experienced values.

1095 Participants were able to infer the value of ingredients based on schematic knowledge

1096 without feedback, and continued showing a benefit from recipe information in subsequent

1097 presentations. Our computational model captured this behavior using normative

1098 information about ingredient-recipe associations. Notably, freely fitting the number of

1099 dimensions used in reconstructing these recipe-ingredient associations improved the fit of 
1100 the model, implying that participants varied in the depth of semantic knowledge they

1101 employed in this task. The positive recipe also had a greater influence on behavior compared

1102 to the negative recipe, indicating that participants prioritized searching associations with

1103 this recipe first - possibly because they adopted a default response to pass on ingredients

1104 where they could not retrieve positive value information. Variance in how participants

1105 engage with these schemas to form value estimates may also be reflected in neural activity,

1106 however we did not have a sufficient sample size to make a strong test of such individual

1107 differences.

1108 Memory strength, expressed as unfamiliarity, had a substantial impact on

1109 participants' willingness to endorse ingredients. Participants demonstrated a general

1110 tendency against betting on independent ingredients on the first presentation and against

1111 betting on ingredients where memory strength was lower on subsequent presentations.

1112 Given that there was an equal proportion of positive and negative value ingredients, and a

$11132: 1$ ratio of points gained to lost on a bet, this behavior reflects a deviation from a rational

1114 maximization of expected value, akin to loss aversion (Kahneman \& Tversky, 1979). While

1115 the proportion of positive to negative value trials was not made explicit to the participants,

1116 and we cannot be sure that participants were aware of this equal weighting, this proportion

1117 was the same in the practice phase and main experiment, so that participants would have

1118 had experience with an equal proportion of positive and negative trials before starting the

1119 main task. Whereas uncertainty is often operationalized as descriptive or experienced

1120 probabilities of outcomes in behavioral economics, here it depended on the strength of

1121 participants' memory of outcomes. These results are also consistent with work showing a

1122 bias toward remembered items in preference-based choice (Gluth, Sommer, Rieskamp, \& 
1123 Buchel, 2015). In real terms, people appear to apply a meta-cognitive strategy where the

1124 strength of evidence from memory affects option value, regardless of its content. We would

1125 thus predict that a strong memory for a weakly positive or negative value would be more

1126 likely to drive a response than a weak memory for a strongly positive or negative value.

1127 Our results indicate that vmPFC represents values based on multiple memory

1128 sources. These findings add to past work demonstrating that the value function represented

1129 in vmPFC can flexibly bend to accommodate attentional priority toward different option

1130 attributes (Hare, Malmaud, \& Rangel, 2011; Hunt, Dolan, \& Behrens, 2014; Leong, Radulescu,

1131 Daniel, DeWoskin, \& Niv, 2017; Rudorf \& Hare, 2014). However, we cannot be sure if the

1132 values represented here are ultimately referenced to the points earned by the participant, or

1133 reflect a social value based on the experience of the "customer" whom they are ostensibly

1134 feeding according to the cover story for the task.

1135 We had predicted that vmPFC would be engaged more by schema-based value

1136 inference that retrieval of a specific experience. This prediction was based on lesion evidence

1137 demonstrating that vmPFC is not necessary for value judgment per se (Reber et al., 2017;

1138 Vaidya \& Fellows, 2015; Yu, Dana, \& Kable, 2018), but is needed for incorporating higher-

1139 order information into value judgments (Vaidya, Sefranek, \& Fellows, 2017; Xia, Stolle,

1140 Gidengil, \& Fellows, 2015). Other work has shown that inactivation or damage to vmPFC or

1141 OFC does not affect decision-making or value judgment in situations where values can be

1142 retrieved from prior direct experience, but does so when this information must be inferred

1143 from structured knowledge about a task (Bradfield et al., 2015; Izquierdo et al., 2004; Jones

1144 et al., 2012; Rudebeck, Saunders, Prescott, Chau, \& Murray, 2013). 
There may be a few factors contributing to this discrepancy. While experienced

1146 values were learned over many repeated exposures in these lesion experiments, here they

1147 were learned from just one or two trials. Recent work has shown that spaced, brief training

1148 over a period of days in a reinforcement learning task increases fMRI value signals in vmPFC

1149 compared to an equivalent number of trials within a single session (Wimmer, Li,

1150 Gorgolewski, \& Poldrack, 2018), implying that this region is engaged more when value

1151 judgments rely on episodic memory rather than massed experience. Although our results

1152 suggest the involvement of vmPFC in representing both directly experienced and schema-

1153 based values, this information may be robustly represented by other regions that do not

1154 depend on vmPFC to influence behavior. Studying the effects of vmPFC damage in this task

1155 could help assess when this region is critical for memory-based value judgment.

1156 Our hypotheses are not fully committed to the precise format of experienced values

1157 in this task. Our design required that participants store information about feedback paired

1158 with ingredients in a small number of trials as a specific experience separated by other trials

1159 over a long interval. We assumed that participants would mostly rely on episodic memory

1160 for robust retention of such information. However, we cannot rule out the possibility that

1161 value information would also be stored in other ways (e.g. in working memory, or through

1162 conditioned stimulus-response mappings). Nonetheless, this possibility does not change our

1163 ability to contrast inference of values from a memory schema and reliance on direct

1164 experience more generally.

1165 Notably, our results indicate that interactions between vmPFC and MTC support

1166 inference of values from semantic knowledge. Other work has shown that utilization of

1167 semantic meaning in value judgments increases interactions between vmPFC and regions of 
1168 left middle and superior temporal cortex (Lim, O'Doherty, \& Rangel, 2013). Here, vmPFC-

1169 MTC connectivity depended on the value of schematic associations. This result could reflect

1170 the accumulation of value information through the interaction between these regions, or the

1171 differential weight given to positive and negative recipes, as there was behavioral evidence

1172 that participants prioritized positive recipe associations. In either case, these data provide

1173 evidence that engagement between vmPFC and semantic memory stores is involved in

1174 inferential value judgments based on real-world structured knowledge in absence of explicit

1175 experienced values.

1176 This experiment explicitly crossed two features commonly associated with the

1177 vmPFC, but rarely compared within the same task: value coding and schema-based memory

1178 and inference (Chan, Niv, \& Norman, 2016; Delgado et al., 2016; Ghosh, Moscovitch, Melo

1179 Colella, \& Gilboa, 2014). Our data are consistent with both proposed roles for this region:

1180 with schema-related knowledge playing a role in value construction in vmPFC, and with this

1181 region responding to utilization of schema-related knowledge more generally. Recent rodent

1182 electrophysiological data also indicate that value and schematic task representations co-

1183 exist in separable codes within OFC (Zhou et al., 2019), suggesting these functions may co-

1184 exist within the same regions.

1185 The role of striatum in memory tasks has been the focus of recent research, with

1186 several hypotheses being considered (Elward et al., 2015; King, Chastelaine, Elward, Wang,

1187 \& Rugg, 2018; Schwarze, Bingel, Badre, \& Sommer, 2013; Scimeca \& Badre, 2012). Here,

1188 activity in the striatum integrated the influence of memory strength on participants'

1189 decisions to bet or pass on ingredients, as its activity correlated with the interaction of

1190 participant-P(yes) and unfamiliarity. Relatedly, recent work from our group has observed 
1191 that the striatum tracked prediction errors for retrieval success during a recognition

1192 memory task (Scimeca et al., 2016), analogous to its role in action selection and

1193 reinforcement-learning (Garrison, Erdeniz, \& Done, 2013; Haber \& Knutson, 2010;

1194 Pessiglione, Seymour, Flandin, Dolan, \& Frith, 2006). These prediction errors were

1195 correlated with changes in the criterion by which evidence from memory was evaluated.

1196 Unlike conventional reinforcement learning settings, the striatum was sensitive to

1197 prediction errors that derived from memory strength rather than the value of a response.

1198 We expand on those findings here, demonstrating that the striatum responds to the influence

1199 of memory strength on participants' willingness to make a bet in a value-based decision-

1200 making task, independent of memory valence. Together, these results imply a role for the

1201 striatum in a cognitive decision about when to act based on memory strength, equivalent to

1202 its role in controlling a tendency to bet in risky decision-making (Knutson, Wimmer, Kuhnen,

1203 \& Winkielman, 2008; Nachev et al., 2015; Zalocusky et al., 2016), or in gating working

1204 memory (Chatham \& Badre, 2015).

1205 Notably, the striatum responded to the strength of memory for experienced values

1206 rather than the experienced values of options themselves. The striatum responds to

1207 successful memory retrieval in many tasks (Spaniol et al., 2009), though the functional

1208 significance of this activation is debated. Some work has argued that these signals reflect a

1209 value signal that depends on the congruence of the memory task with participants' goals

1210 (Han et al., 2010), while others have suggested that striatal reward and retrieval signals are

1211 distinct (Elward et al., 2015). Here we found evidence that unsigned memory strength (i.e.

1212 unfamiliarity) modulates striatal value signals, but the signed experienced value of options

1213 did not. These data indicate that value and retrieval signals in the striatum may follow a 
1214 common dimension that corresponds to the utility of retrieved information given task

1215 demands - biasing cortical representations of associated actions based on the strength of

1216 information recovered from retrieval.

1217 We also found that the striatum responded to the interaction of participant-P(yes)

1218 and schema value. While of a smaller effect size, these data imply that the striatum encodes

1219 these inferred values in addition to the effects of unfamiliarity. Indeed, previous work has

1220 found that the striatum responds to prediction errors inferred structured knowledge about

1221 a task (Daw, Gershman, Seymour, Dayan, \& Dolan, 2011; Lohrenz, McCabe, Camerer, \&

1222 Montague, 2007).

1223 We also observed that connectivity between HPC and the striatum increased in the

1224 second and third presentations of independent ingredients compared to recipe ingredients,

1225 and on correct choices for independent ingredients compared to error trials. This result

1226 implies that information shared in a HPC-striatal circuit is involved in successful retrieval of

1227 experienced ingredient-value associations. While this result depended on the choice of seed

1228 ROI, this apparent selectivity might owe to our striatal ROI averaging over functionally

1229 distinct subregions.

1230 In conclusion, we demonstrated the involvement of the striatum and vmPFC in

1231 distinct processes within memory-based decision-making. Our data provide fresh insights

1232 into the dynamics of this circuitry, with a view into the intersection of decision-making and

1233 memory that is the seat of much real-world choice behavior. These results have implications

1234 for understanding the origins of decision-making impairments in neurodegenerative

1235 diseases such as Alzheimer's disease and frontotemporal dementia (Salmon \& Bondi, 2009),

1236 as well as Parkinson's disease (Edelstyn, Shepherd, Mayes, Sherman, \& Ellis, 2010). New 
bioRxiv preprint doi: https://doi.org/10.1101/712661; this version posted April 22, 2020. The copyright holder for this preprint (which was not certified by peer review) is the author/funder, who has granted bioRxiv a license to display the preprint in perpetuity. It is made available under aCC-BY-NC-ND 4.0 International license.

1237 insights into the interaction of these circuits may have relevance for clinical interventions or 1238 treatments.

1239 


\section{References}

1241 Badre, D., Poldrack, R. A., Pare-Blagoev, E. J., Insler, R. Z., \& Wagner, A. D. (2005a). Dissociable controlled retrieval and generalized selection mechanisms in ventrolateral prefrontal cortex. Neuron, 47(6), 907-918.

1243

1244 doi:10.1016/j.neuron.2005.07.023

Badre, D., Poldrack, R. A., Pare-Blagoev, E. J., Insler, R. Z., \& Wagner, A. D. (2005b). Dissociable controlled retrieval and generalized selection mechanisms in ventrolateral prefrontal cortex. Neuron, 47(6), 907-918. doi:10.1016/j.neuron.2005.07.023

Barredo, J., Verstynen, T. D., \& Badre, D. (2016). Organization of cortico-cortical pathways supporting memory retrieval across subregions of the left ventrolateral prefrontal cortex. J Neurophysiol, 116(3), 920-937. doi:10.1152/jn.00157.2016

Barron, H. C., Dolan, R. J., \& Behrens, T. E. (2013). Online evaluation of novel choices by simultaneous representation of multiple memories. Nat Neurosci, 16(10), 14921498. doi:10.1038/nn.3515

Bartlett, F. C. (1932). Remembering: A Study in Experimental and Social Psychology: Cambridge University Press.

Bartra, O., McGuire, J. T., \& Kable, J. W. (2013). The valuation system: a coordinate-based meta-analysis of BOLD fMRI experiments examining neural correlates of subjective value. Neuroimage, 76, 412-427. doi:10.1016/j.neuroimage.2013.02.063

Blechert, J., Meule, A., Busch, N. A., \& Ohla, K. (2014). Food-pics: an image database for experimental research on eating and appetite. Front Psychol, 5, 617. doi:10.3389/fpsyg.2014.00617

Bradfield, L. A., Dezfouli, A., van Holstein, M., Chieng, B., \& Balleine, B. W. (2015). Medial Orbitofrontal Cortex Mediates Outcome Retrieval in Partially Observable Task Situations. Neuron, 88(6), 1268-1280. doi:10.1016/j.neuron.2015.10.044

Braga, R. M., \& Buckner, R. L. (2017). Parallel Interdigitated Distributed Networks within the Individual Estimated by Intrinsic Functional Connectivity. Neuron, 95(2), 457471 e455. doi:10.1016/j.neuron.2017.06.038

Brainard, D. H. (1997). The psychophysics toolbox. Spatial Vision, 10(4), 433-436. doi:Doi $10.1163 / 156856897 \times 00357$

Brett, M., Anton, J., Valabregue, R., \& Poline, J. (2002). Region of interest analysis using an SPM toolbox. Paper presented at the 8th International Conference on Functional Mapping of the Human Brain, Sendai, Japan.

Brodeur, M. B., Dionne-Dostie, E., Montreuil, T., \& Lepage, M. (2010). The Bank of Standardized Stimuli (BOSS), a new set of 480 normative photos of objects to be used as visual stimuli in cognitive research. PLoS One, 5(5), e10773. doi:10.1371/journal.pone.0010773

Brodeur, M. B., Guerard, K., \& Bouras, M. (2014). Bank of Standardized Stimuli (BOSS) phase II: 930 new normative photos. PLoS One, 9(9), e106953. doi:10.1371/journal.pone.0106953

Chan, S. C. Y., Niv, Y., \& Norman, K. A. (2016). A Probability Distribution over Latent Causes, in the Orbitofrontal Cortex. Journal of Neuroscience, 36(30), 7817-7828. doi:10.1523/JNEUROSCI.0659-16.2016 
1284 Chatham, C. H., \& Badre, D. (2015). Multiple gates on working memory. Curr Opin Behav Sci, 1, 23-31. doi:10.1016/j.cobeha.2014.08.001 influences on humans' choices and striatal prediction errors. Neuron, 69(6), 12041215. doi:10.1016/j.neuron.2011.02.027

Delgado, M. R., Beer, J. S., Fellows, L. K., Huettel, S. A., Platt, M. L., Quirk, G. J., \& Schiller, D. (2016). Viewpoints: Dialogues on the functional role of the ventromedial prefrontal cortex. Nat Neurosci, 19(12), 1545-1552. doi:10.1038/nn.4438

Dumais, S. T. (2004). Latent semantic analysis. Annual Review of Information Science and Technology, 38, 189-230.

Edelstyn, N. M., Shepherd, T. A., Mayes, A. R., Sherman, S. M., \& Ellis, S. J. (2010). Effect of disease severity and dopaminergic medication on recollection and familiarity in patients with idiopathic nondementing Parkinson's. Neuropsychologia, 48(5), 13671375. doi:10.1016/j.neuropsychologia.2009.12.039

Eichenbaum, H. (2017). Memory: Organization and Control. Annu Rev Psychol, 68, 19-45. doi:10.1146/annurev-psych-010416-044131

Elward, R. L., Vilberg, K. L., \& Rugg, M. D. (2015). Motivated Memories: Effects of Reward and Recollection in the Core Recollection Network and Beyond. Cereb Cortex, 25(9), 3159-3166. doi:10.1093/cercor/bhu109

Garrison, J., Erdeniz, B., \& Done, J. (2013). Prediction error in reinforcement learning: a meta-analysis of neuroimaging studies. Neurosci Biobehav Rev, 37(7), 1297-1310. doi:10.1016/j.neubiorev.2013.03.023

Ghosh, V. E., \& Gilboa, A. (2014). What is a memory schema? A historical perspective on current neuroscience literature. Neuropsychologia, 53, 104-114. doi:10.1016/j.neuropsychologia.2013.11.010

Ghosh, V. E., Moscovitch, M., Melo Colella, B., \& Gilboa, A. (2014). Schema representation in patients with ventromedial PFC lesions. J Neurosci, 34(36), 12057-12070. doi:10.1523/JNEUROSCI.0740-14.2014

Gluth, S., Sommer, T., Rieskamp, J., \& Buchel, C. (2015). Effective Connectivity between Hippocampus and Ventromedial Prefrontal Cortex Controls Preferential Choices from Memory. Neuron, 86(4), 1078-1090. doi:10.1016/j.neuron.2015.04.023

Haber, S. N., \& Knutson, B. (2010). The reward circuit: linking primate anatomy and human imaging. Neuropsychopharmacology, 35(1), 4-26. doi:10.1038/npp.2009.129

Han, S., Huettel, S. A., Raposo, A., Adcock, R. A., \& Dobbins, I. G. (2010). Functional Significance of Striatal Responses during Episodic Decisions: Recovery or Goal Attainment? Journal of Neuroscience, 30(13), 4767-4775. doi:10.1523/Jneurosci.3077-09.2010

Hare, T. A., Malmaud, J., \& Rangel, A. (2011). Focusing attention on the health aspects of foods changes value signals in vmPFC and improves dietary choice. J Neurosci, 31(30), 11077-11087. doi:10.1523/JNEUROSCI.6383-10.2011

Hunt, L. T., Dolan, R. J., \& Behrens, T. E. (2014). Hierarchical competitions subserving multiattribute choice. Nat Neurosci, 17(11), 1613-1622. doi:10.1038/nn.3836

Izquierdo, A., Suda, R. K., \& Murray, E. A. (2004). Bilateral orbital prefrontal cortex lesions in rhesus monkeys disrupt choices guided by both reward value and reward contingency. J Neurosci, 24(34), 7540-7548. doi:10.1523/JNEUROSCI.1921-04.2004 
1329

1330

1331

1332

1333

1334

1335

1336

1337

1338

1339

1340

1341

1342

1343

1344

1345

1346

1347

1348

1349

1350

1351

1352

1353

1354

1355

1356

1357

1358

1359

1360

1361

1362

1363

1364

1365

1366

1367

1368

1369

1370

1371

1372

1373
Jones, J. L., Esber, G. R., McDannald, M. A., Gruber, A. J., Hernandez, A., Mirenzi, A., \& Schoenbaum, G. (2012). Orbitofrontal Cortex Supports Behavior and Learning Using Inferred But Not Cached Values. Science, 338(6109), 953-956. doi:Doi 10.1126/Science.1227489

Kahneman, D., \& Tversky, A. (1979). Prospect Theory - Analysis of Decision under Risk. Econometrica, 47(2), 263-291. doi:Doi 10.2307/1914185

King, D. R., Chastelaine, M., Elward, R. L., Wang, T. H., \& Rugg, M. D. (2018). Dissociation between the neural correlates of recollection and familiarity in the striatum and hippocampus: Across-study convergence. Behav Brain Res, 354, 1-7. doi:10.1016/j.bbr.2017.07.031

Kleiner, M., Brainard, D., \& Pelli, D. (2007). What's new in Psychtoolbox-3? Perception, 36, 14-14.

Knutson, B., Wimmer, G. E., Kuhnen, C. M., \& Winkielman, P. (2008). Nucleus accumbens activation mediates the influence of reward cues on financial risk taking. Neuroreport, 19(5), 509-513. doi:10.1097/WNR.0b013e3282f85c01

Landauer, T. K., Foltz, P. W., \& Laham, D. (1998). An introduction to latent semantic analysis. Discourse Processes, 25(2-3), 259-284. doi:Doi $10.1080 / 01638539809545028$

Leong, Y. C., Radulescu, A., Daniel, R., DeWoskin, V., \& Niv, Y. (2017). Dynamic Interaction between Reinforcement Learning and Attention in Multidimensional Environments. Neuron, 93(2), 451-463. doi:10.1016/j.neuron.2016.12.040

Lim, S. L., O'Doherty, J. P., \& Rangel, A. (2013). Stimulus value signals in ventromedial PFC reflect the integration of attribute value signals computed in fusiform gyrus and posterior superior temporal gyrus. J Neurosci, 33(20), 8729-8741. doi:10.1523/JNEUROSCI.4809-12.2013

Lohrenz, T., McCabe, K., Camerer, C. F., \& Montague, P. R. (2007). Neural signature of fictive learning signals in a sequential investment task. Proc Natl Acad Sci U S A, 104(22), 9493-9498. doi:10.1073/pnas.0608842104

McLaren, D. G., Ries, M. L., Xu, G., \& Johnson, S. C. (2012). A generalized form of contextdependent psychophysiological interactions (gPPI): a comparison to standard approaches. Neuroimage, 61(4), 1277-1286. doi:10.1016/j.neuroimage.2012.03.068

Nachev, P., Lopez-Sosa, F., Gonzalez-Rosa, J. J., Galarza, A., Avecillas, J., Pineda-Pardo, J. A., . . . Strange, B. (2015). Dynamic risk control by human nucleus accumbens. Brain, 138, 3496-3502. doi:10.1093/brain/awv285

Pessiglione, M., Seymour, B., Flandin, G., Dolan, R. J., \& Frith, C. D. (2006). Dopaminedependent prediction errors underpin reward-seeking behaviour in humans. Nature, 442(7106), 1042-1045. doi:10.1038/nature05051

Piaget, J. (1952). The Origins of Intelligence in Children (Vol. 8). International Universities Press.

Postman, L., \& Keppel, G. (1970). Norms of Word Asociation. New York, New York: Academic Press.

Price, J. L. (2007). Definition of the orbital cortex in relation to specific connections with limbic and visceral structures and other cortical regions. Ann N Y Acad Sci, 1121, 5471. doi:annals.1401.008 [pii]

10.1196/annals.1401.008 
1374 Ratcliff, R. (1978). Theory of Memory Retrieval. Psychological Review, 85(2), 59-108.

1375

1376

1377

1378

1379

1380

1381

1382

1383

1384

1385

1386

1387

1388

1389

1390

1391

1392

1393

1394

1395

1396

1397

1398

1399

1400

1401

1402

1403

1404

1405

1406

1407

1408

1409

1410

1411

1412

1413

1414

1415

1416

1417

1418 doi:Doi 10.1037//0033-295x.85.2.59

Reber, J., Feinstein, J. S., O'Doherty, J. P., Liljeholm, M., Adolphs, R., \& Tranel, D. (2017). Selective impairment of goal-directed decision-making following lesions to the human ventromedial prefrontal cortex. Brain, 140(6), 1743-1756. doi:10.1093/brain/awx105

Rescorla, R. A., \& Wagner, A. R. (1972). A theory of Pavlovian conditioning: Variations in the effective- ness of reinforcement and non-reinforcement. In A. H. Black \& W. F. Prokasy (Eds.), Classical conditioning. II. Current research and theory (pp. 64-99). New York: Appleton-Century-Crofts.

Roy, M., Shohamy, D., \& Wager, T. D. (2012). Ventromedial prefrontal-subcortical systems and the generation of affective meaning. Trends Cogn Sci, 16(3), 147-156. doi:10.1016/j.tics.2012.01.005

Rudebeck, P. H., Saunders, R. C., Prescott, A. T., Chau, L. S., \& Murray, E. A. (2013). Prefrontal mechanisms of behavioral flexibility, emotion regulation and value updating. Nat Neurosci, 16(8), 1140-1145. doi:10.1038/nn.3440

Rudorf, S., \& Hare, T. A. (2014). Interactions between Dorsolateral and Ventromedial Prefrontal Cortex Underlie Context-Dependent Stimulus Valuation in Goal-Directed Choice. Journal of Neuroscience, 34(48), 15988-15996. doi:10.1523/JNEUROSCI.3192-14.2014

Saleem, K. S., Kondo, H., \& Price, J. L. (2008). Complementary circuits connecting the orbital and medial prefrontal networks with the temporal, insular, and opercular cortex in the macaque monkey. J Comp Neurol, 506(4), 659-693. doi:10.1002/cne.21577

Salmon, D. P., \& Bondi, M. W. (2009). Neuropsychological assessment of dementia. Annu Rev Psychol, 60, 257-282. doi:10.1146/annurev.psych.57.102904.190024

Schuck, N. W., Cai, M. B., Wilson, R. C., \& Niv, Y. (2016). Human Orbitofrontal Cortex Represents a Cognitive Map of State Space. Neuron, 91(6), 1402-1412. doi:10.1016/j.neuron.2016.08.019

Schwarze, U., Bingel, U., Badre, D., \& Sommer, T. (2013). Ventral striatal activity correlates with memory confidence for old- and new-responses in a difficult recognition test. PLoS One, 8(3), e54324. doi:10.1371/journal.pone.0054324

Scimeca, J. M., \& Badre, D. (2012). Striatal contributions to declarative memory retrieval. Neuron, 75(3), 380-392. doi:10.1016/j.neuron.2012.07.014

Scimeca, J. M., Katzman, P. L., \& Badre, D. (2016). Striatal prediction errors support dynamic control of declarative memory decisions. Nat Commun, 7, 13061. doi:10.1038/ncomms13061

Shadlen, M. N., \& Shohamy, D. (2016). Decision Making and Sequential Sampling from Memory. Neuron, 90(5), 927-939. doi:10.1016/j.neuron.2016.04.036

Spaniol, J., Davidson, P. S., Kim, A. S., Han, H., Moscovitch, M., \& Grady, C. L. (2009). Eventrelated fMRI studies of episodic encoding and retrieval: meta-analyses using activation likelihood estimation. Neuropsychologia, 47(8-9), 1765-1779. doi:10.1016/j.neuropsychologia.2009.02.028

Thompson-Schill, S. L., D'Esposito, M., Aguirre, G. K., \& Farah, M. J. (1997). Role of left inferior prefrontal cortex in retrieval of semantic knowledge: a reevaluation. Proc Natl Acad Sci U S A, 94(26), 14792-14797. 
1419

1420

1421

1422

1423

1424

1425

1426

1427

1428

1429

1430

1431

1432

1433

1434

1435

1436

1437

1438

1439

1440

1441

1442

1443

1444

1445

1446

1447

1448

1449

1450

1451

1452

1453

1454

1455

1456
Tzourio-Mazoyer, N., Landeau, B., Papathanassiou, D., Crivello, F., Etard, O., Delcroix, N., ... Joliot, M. (2002). Automated anatomical labeling of activations in SPM using a macroscopic anatomical parcellation of the MNI MRI single-subject brain. Neuroimage, 15(1), 273-289. doi:10.1006/nimg.2001.0978

Vaidya, A. R., \& Fellows, L. K. (2015). Testing necessary regional frontal contributions to value assessment and fixation-based updating. Nat Commun, 6, 10120. doi:10.1038/ncomms10120

Vaidya, A. R., Sefranek, M., \& Fellows, L. K. (2017). Ventromedial Frontal Lobe Damage Alters how Specific Attributes are Weighed in Subjective Valuation. Cereb Cortex, 111. doi:10.1093/cercor/bhx246

Whitfield-Gabrieli, S., \& Nieto-Castanon, A. (2012). Conn: a functional connectivity toolbox for correlated and anticorrelated brain networks. Brain Connect, 2(3), 125-141. doi:10.1089/brain.2012.0073

Wilson, R. C., Takahashi, Y. K., Schoenbaum, G., \& Niv, Y. (2014). Orbitofrontal cortex as a cognitive map of task space. Neuron, 81(2), 267-279. doi:10.1016/j.neuron.2013.11.005

Wimmer, G. E., Li, J. M. K., Gorgolewski, K. J., \& Poldrack, R. A. (2018). Reward Learning over Weeks Versus Minutes Increases the Neural Representation of Value in the Human Brain. Journal of Neuroscience, 38(35), 7649-7666. doi:10.1523/Jneurosci.007518.2018

Xia, C., Stolle, D., Gidengil, E., \& Fellows, L. K. (2015). Lateral orbitofrontal cortex links social impressions to political choices. J Neurosci, 35(22), 8507-8514. doi:10.1523/JNEUROSCI.0526-15.2015

Yonelinas, A. P. (1994). Receiver-Operating Characteristics in Recognition Memory Evidence for a Dual-Process Model. Journal of Experimental Psychology-Learning Memory and Cognition, 20(6), 1341-1354. doi:Doi 10.1037/0278-7393.20.6.1341

Yu, L. Q., Dana, J., \& Kable, J. W. (2018). Individuals with ventromedial frontal damage have more unstable but still fundamentally transitive preferences. bioRxiv, 384024 . doi:10.1101/384024

Zalocusky, K. A., Ramakrishnan, C., Lerner, T. N., Davidson, T. J., Knutson, B., \& Deisseroth, K. (2016). Nucleus accumbens D2R cells signal prior outcomes and control risky decision-making. Nature, 531(7596), 642-646. doi:10.1038/nature17400

Zhou, J., Gardner, M. P. H., Stalnaker, T. A., Ramus, S. J., Wikenheiser, A. M., Niv, Y., \& Schoenbaum, G. (2019). Rat Orbitofrontal Ensemble Activity Contains Multiplexed but Dissociable Representations of Value and Task Structure in an Odor Sequence Task. Curr Biol, 29(6), 897-907 e893. doi:10.1016/j.cub.2019.01.048 


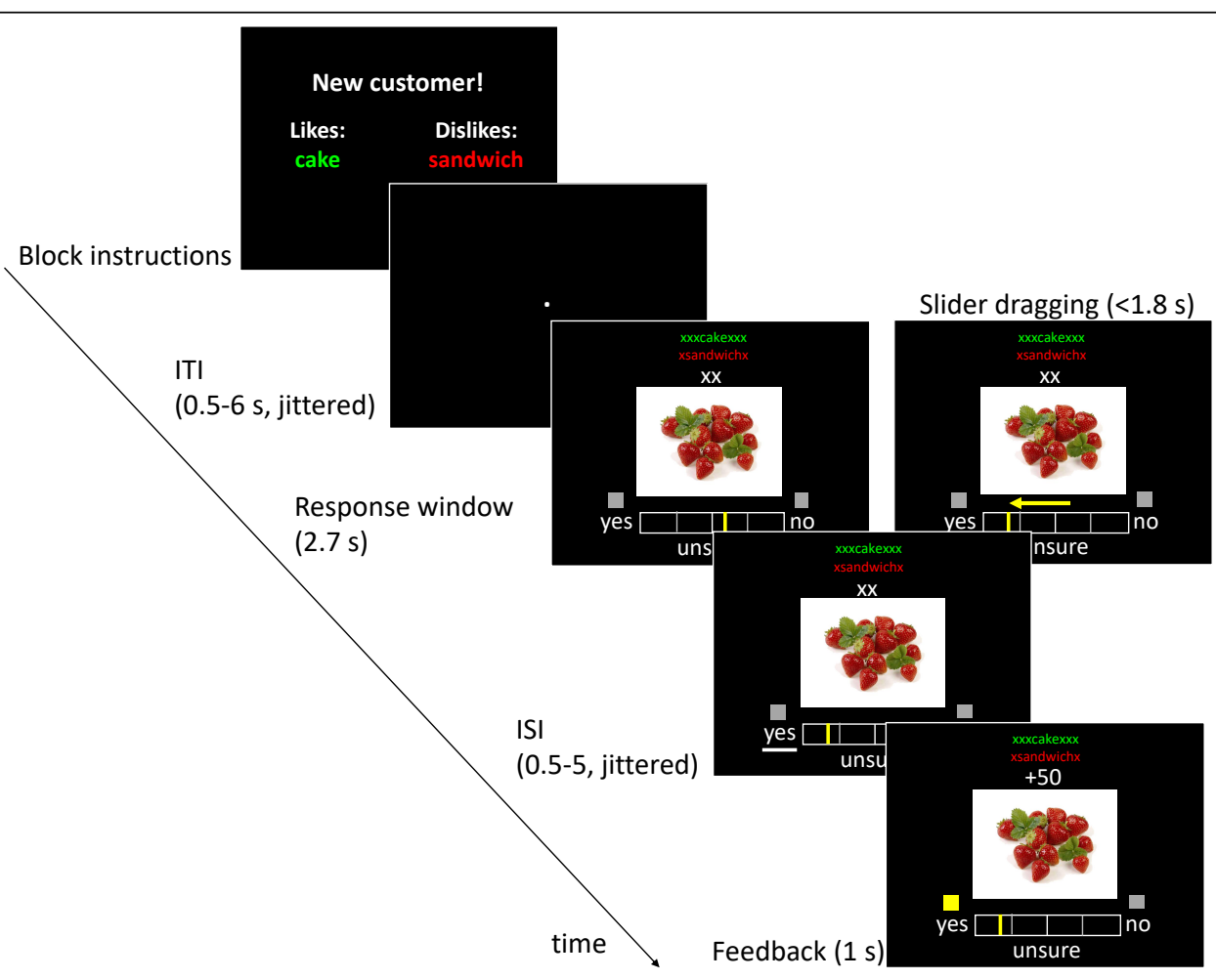

Figure 1. Task schematic showing instructions at the start of a block and organization of a single example trial. At the start of the block, participants were informed of the recipes that customer liked and disliked. These recipe names were presented in green and red above the ingredient on all trials. In each trial, participants indicated their decision to feed the displayed ingredient to the current customer, and their confidence in this decision, using a slider. Participants then saw whether or not the customer liked the ingredient, signaled by a box above either response cue, and the points earned or lost in each trial. ITI (inter-trial interval), ISI (inter-stimulus interval). 
bioRxiv preprint doi: https://doi.org/10.1101/712661; this version posted April 22, 2020. The copyright holder for this preprint (which was not certified by peer review) is the author/funder, who has granted bioRxiv a license to display the preprint in perpetuity. It is made available under aCC-BY-NC-ND 4.0 International license.
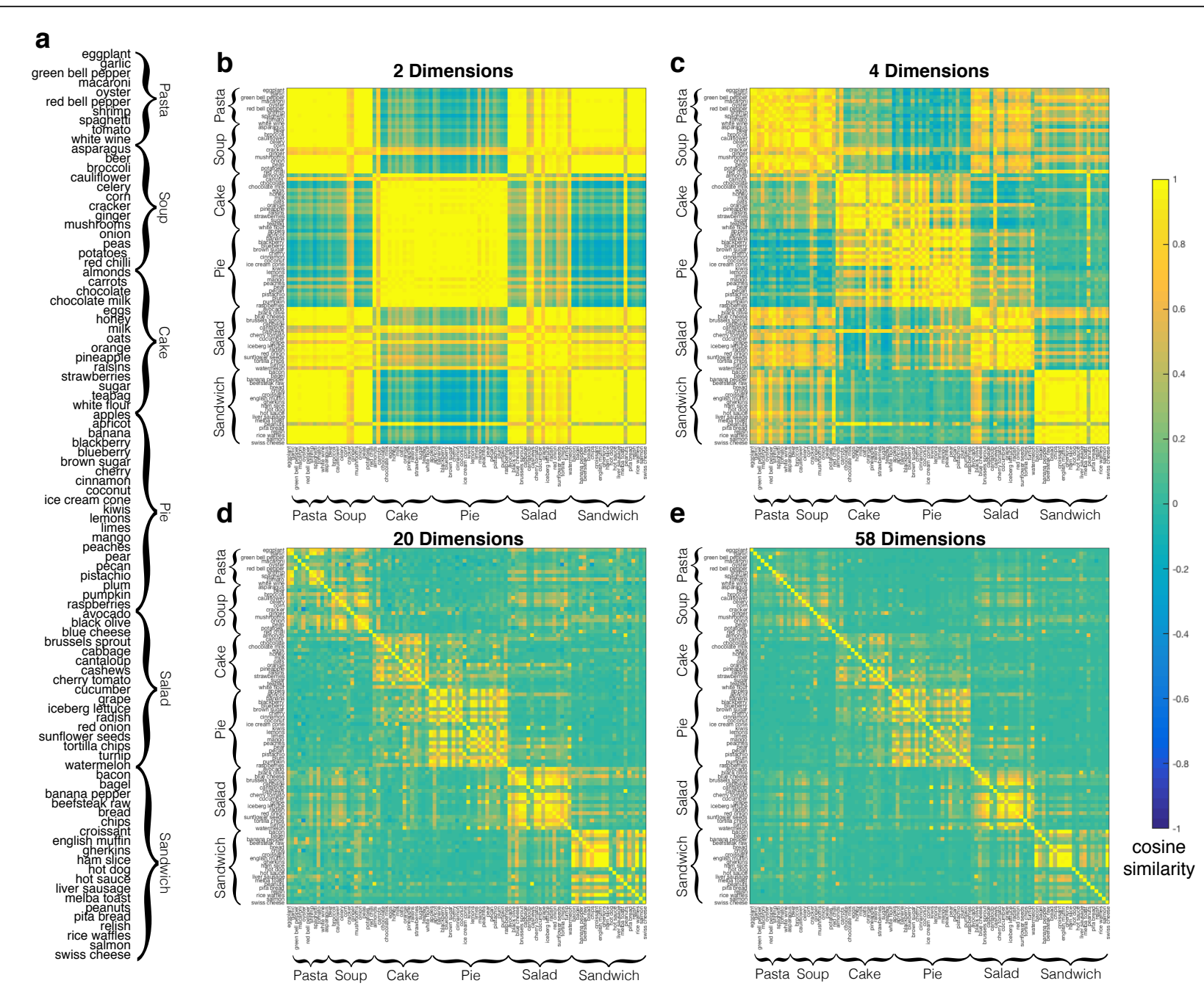

Figure 2. Example ingredient by ingredient cosine similarity matrices from reconstructions of ingredient-recipe associations with increasing dimensions. a. Ingredient names and clustering by recipe. b. Cosine similarity matrix with two dimensions c. with four dimensions, d. with 20 dimensions, and e. with 58 dimensions. 


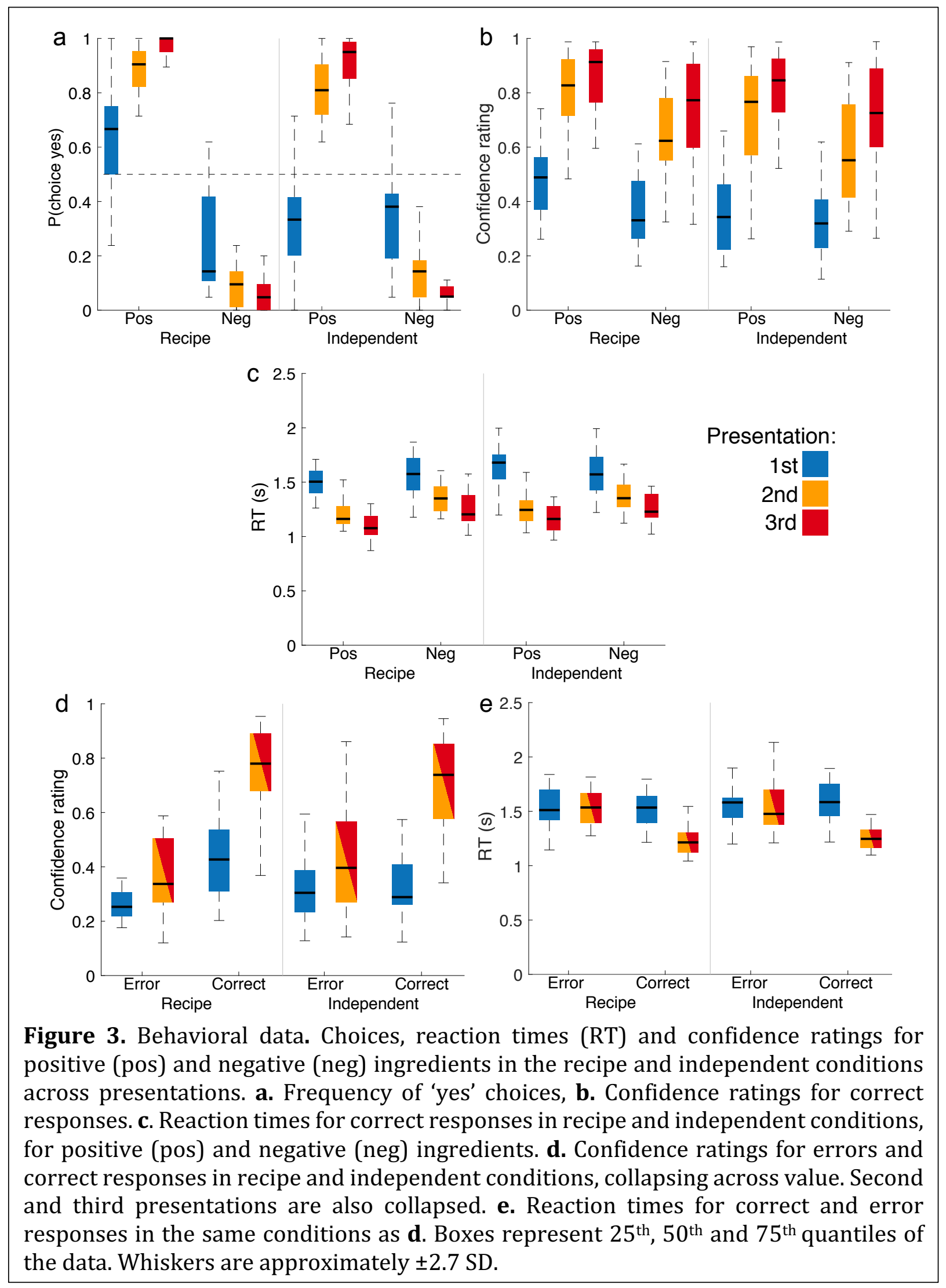


a. Recipe> Independent $\quad k=148$
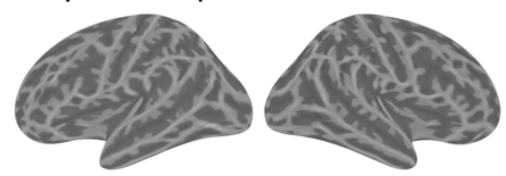

b. Positive $>$ Negative Value $k=157$
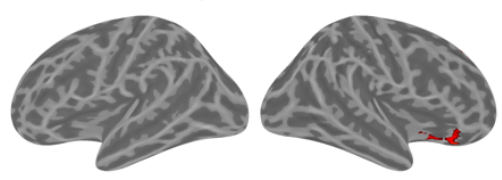

C. Presentation $1>2>3 \quad k=173$
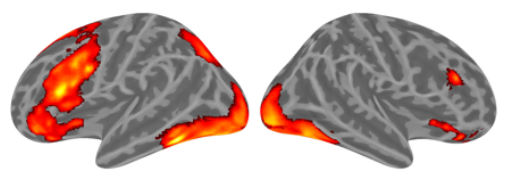

d. Correct $>$ Error $\quad k=210$
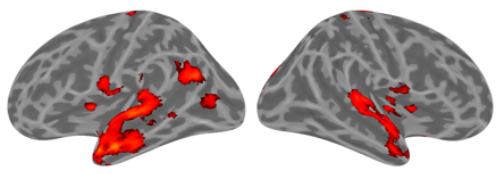
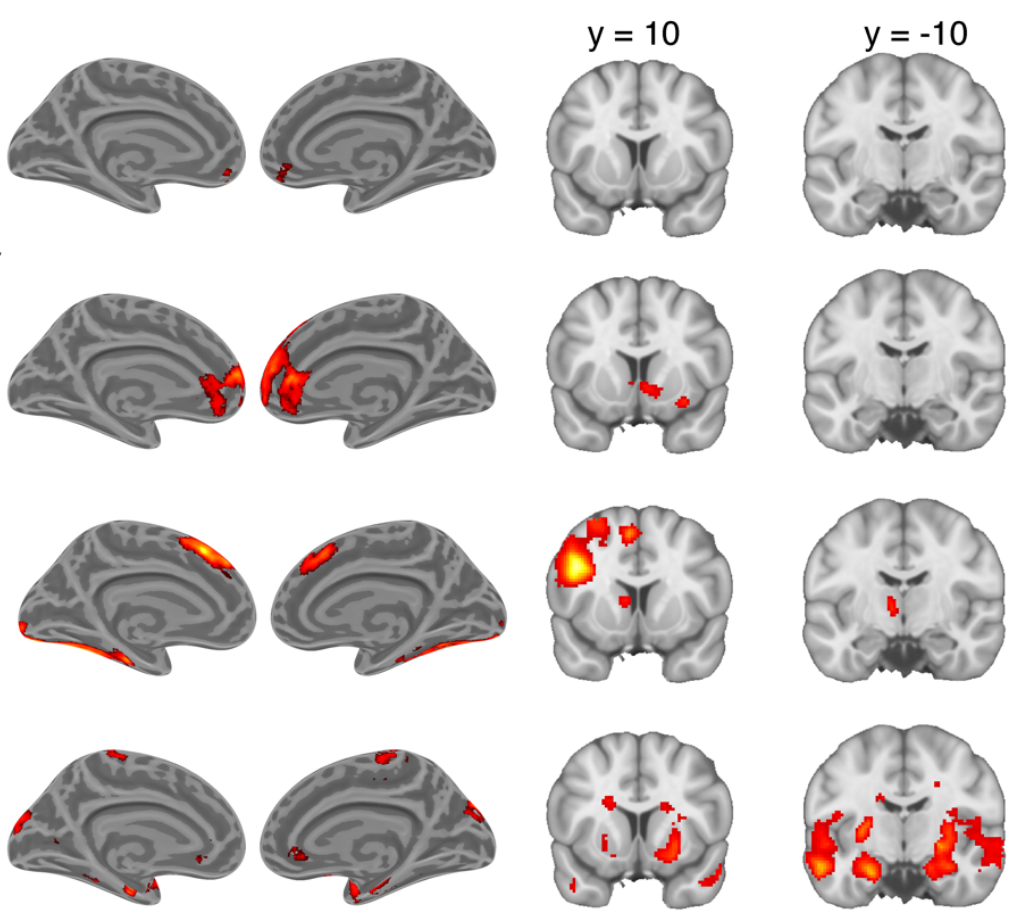

0

t-stat

Figure 4. Whole brain contrasts projected onto inflated cortical surfaces and coronal slices for main effects of $\mathbf{a}$. ingredient-recipe clustering, $\mathbf{b}$. ingredient value, c. presentation number and $\mathbf{d}$. response accuracy. All images are thresholded at $P<0.05$, FWE cluster corrected. All main effects, except for accuracy, are filtered to only include correct responses. Cluster extent threshold for each contrast is given by value of $\mathrm{k}$. 

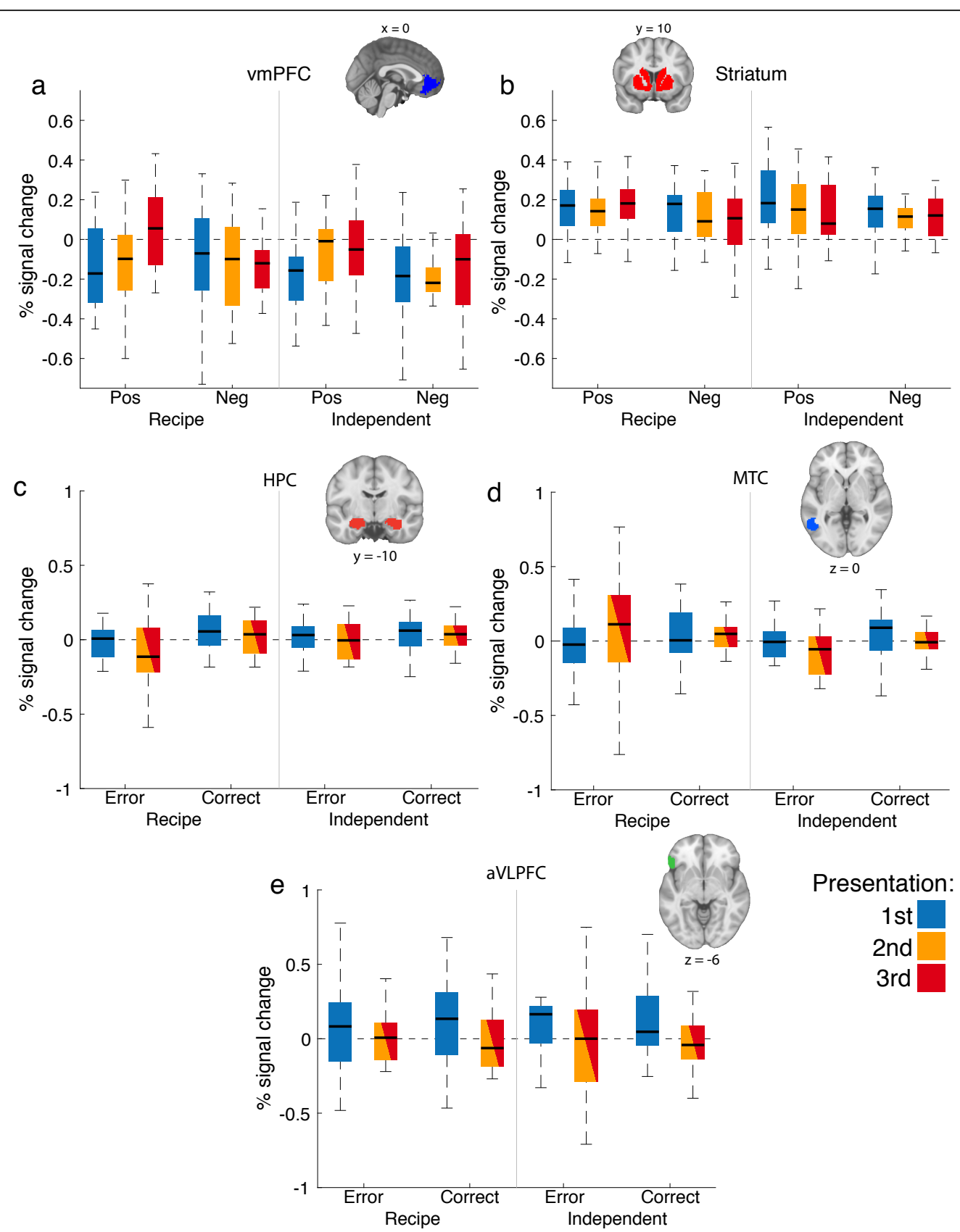

$$
\text { aVLPFC }
$$
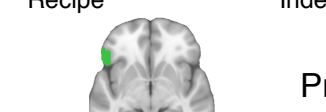

Presentation:

$1 \mathrm{st}$

2nd

3rd

Figure 5. Region-of-interest analyses showing percent signal change in a. ventromedial prefrontal cortex (vmPFC) and $\mathbf{b}$. the bilateral striatum on correct response trials for positive (pos) and negative (neg) ingredients in the recipe and independent conditions across presentations. c. Percent signal change in the hippocampus (HPC) for correct and erroneous responses in the recipe and independent conditions, collapsed across value conditions. Second and third presentations are collapsed. d. Middle temporal cortex (MTC) and e. anterior ventrolateral prefrontal cortex (aVLPFC), same conditions as c. 


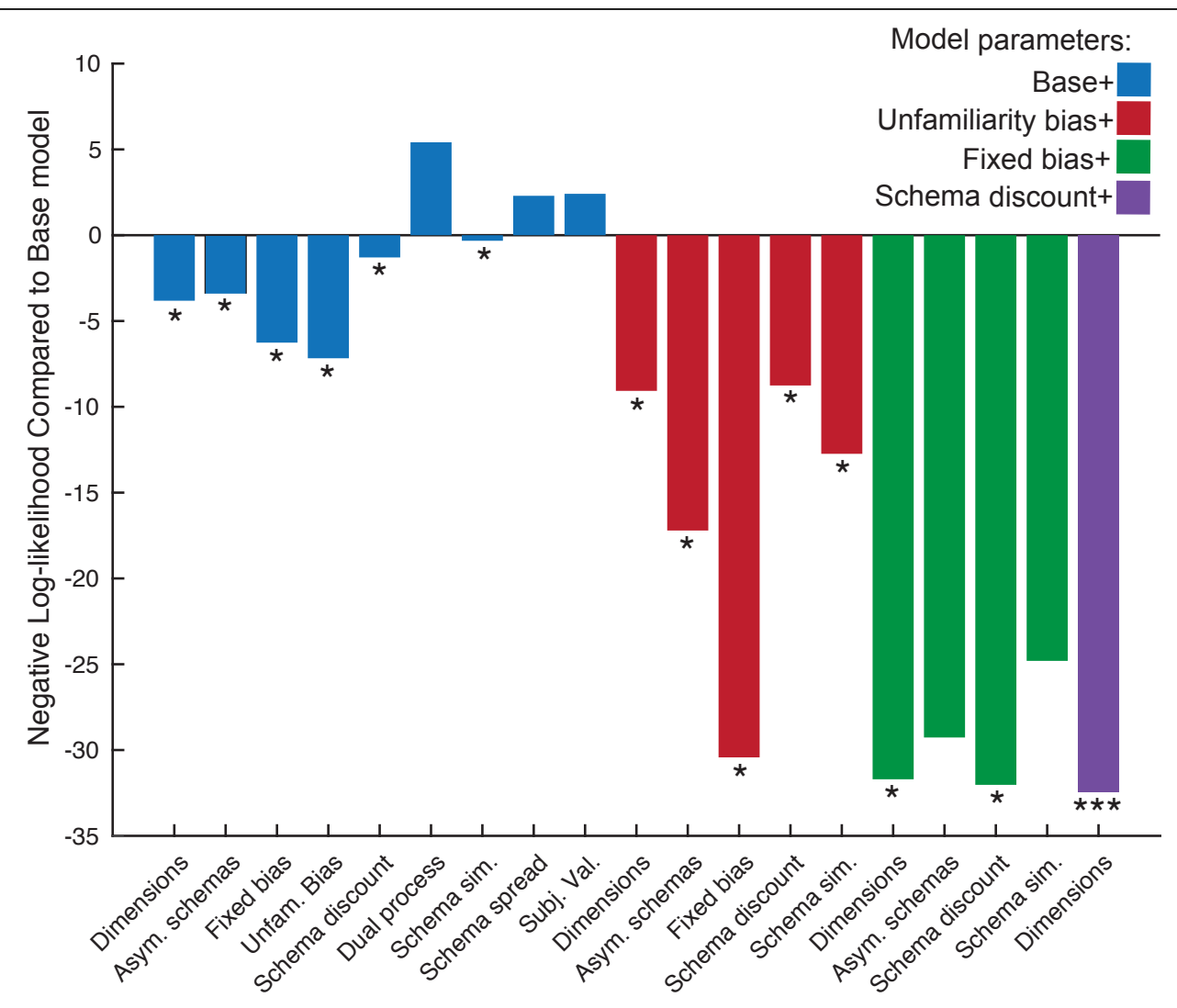

Figure 6. Difference in negative log-likelihood for held-out data in cross-validation procedure for additional model parameters compared to base model. Model parameters were added in a step-wise process where the parameters that best improved on the previous best fitting model were added to the next step (indicated in the legend). See Materials and Methods section for a more complete description of these models. * Reduction in negative log-likelihood compared to next best fitting model. ***, best model within a step and an improvement in fitness compared to next best model. 


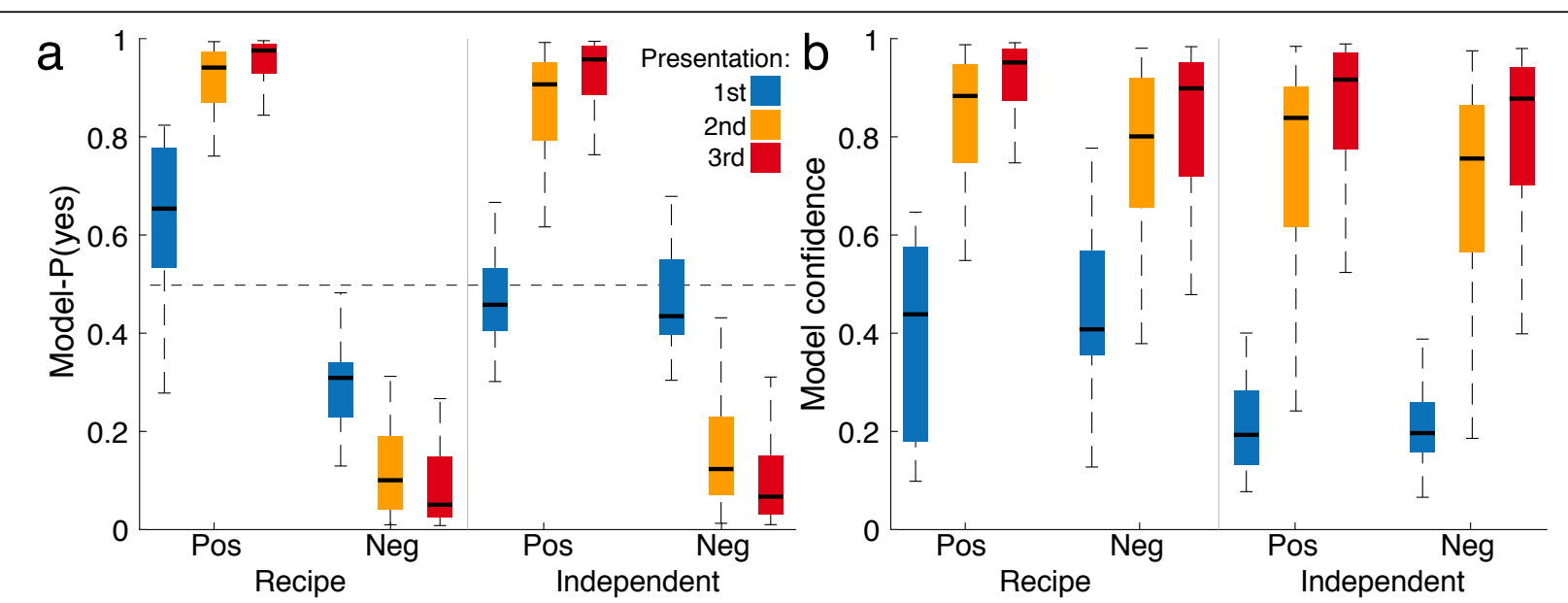

Figure 7. Model behavior for positive (pos) and negative (neg) ingredients in the recipe and independent conditions across presentations. a. Model-P(yes), a continuous indicator of the model's estimate of the value of these options. B. Model confidence on correct responses only. 


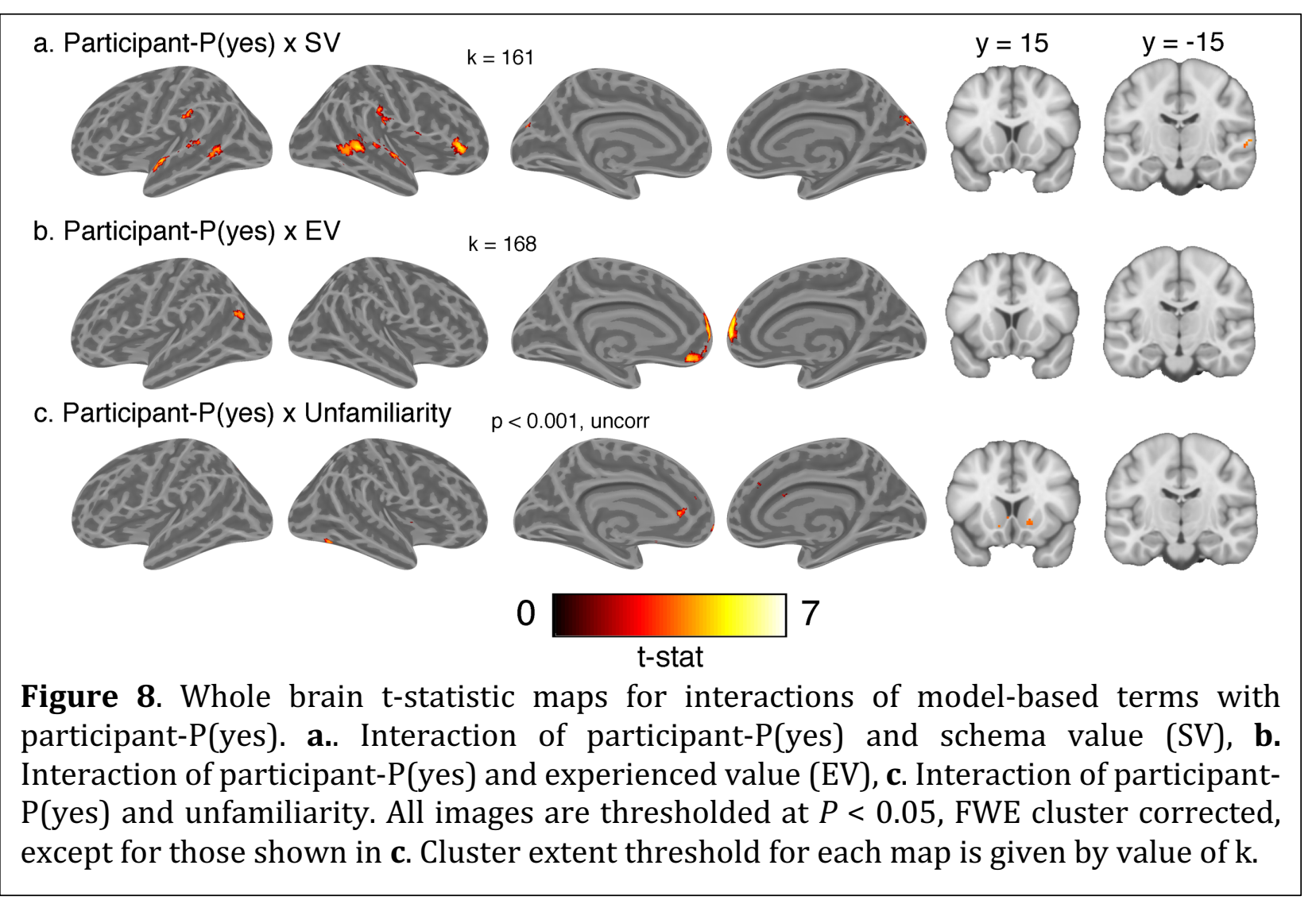




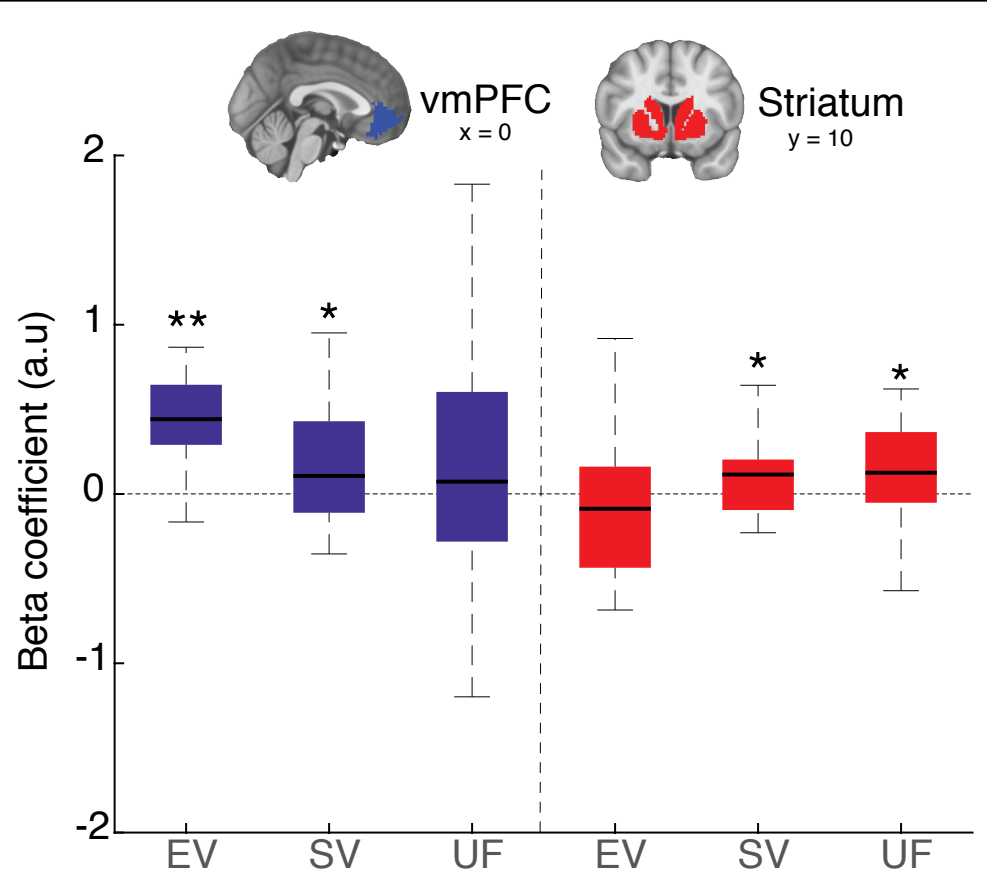

Figure 9. Boxplots showing mean beta coefficients from voxels in ventromedial prefrontal cortex (vmPFC) and striatum ROIs for interactions of participant-P(yes) with experienced value (EV), schema value (SV) and unfamiliarity (UF). ${ }^{* *} P<0.01$, $* P<0.05$, one-tailed one-sample t-test against zero. 


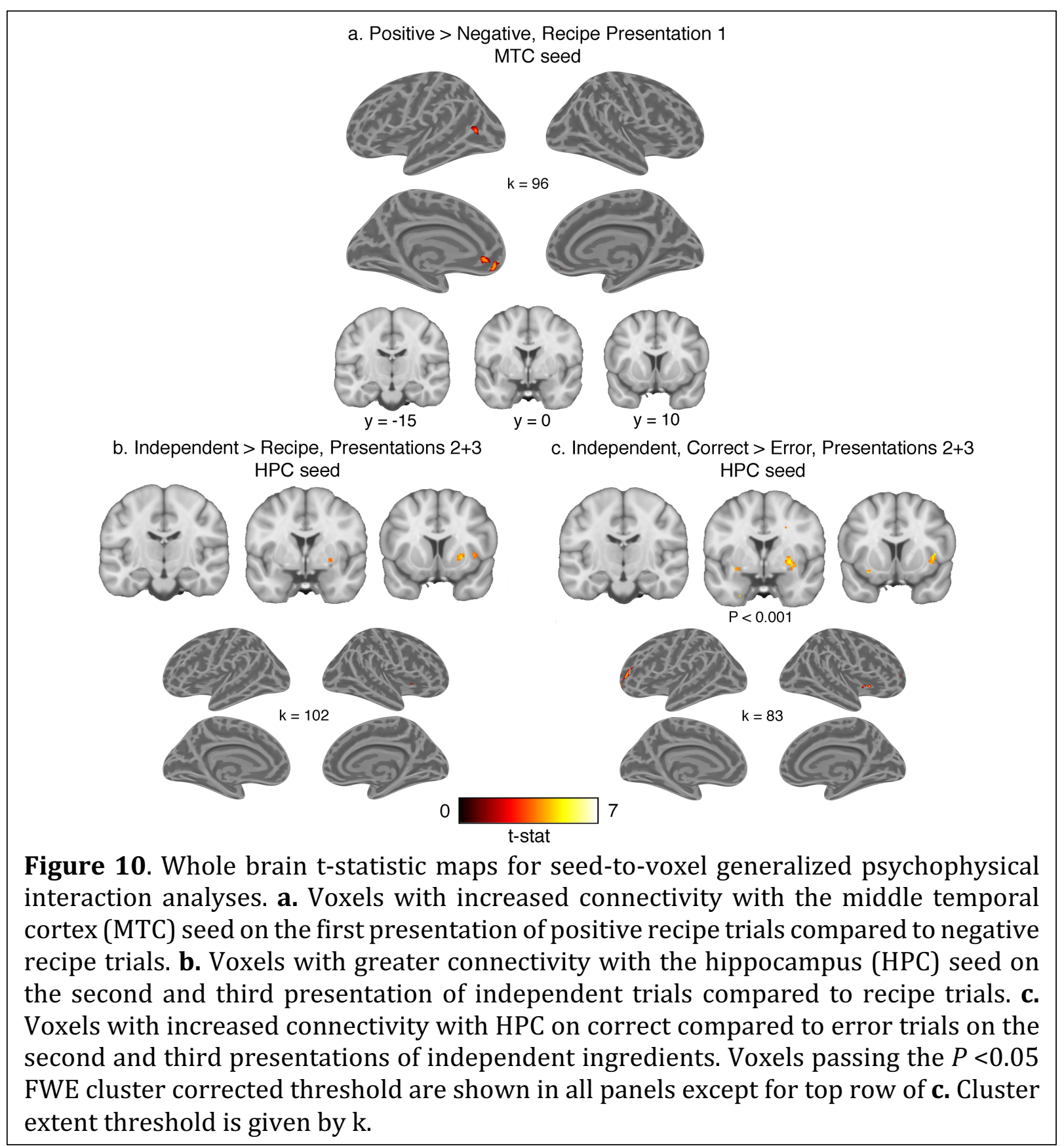


1474 Table 1. Description of ROIs

\begin{tabular}{|l|l|l|}
\hline Region of interest & Definition & Center of mass (MNI) \\
\hline vmPFC & $\begin{array}{l}\text { Positive }>\text { Negative effects of subjective } \\
\text { value (Bartra et al., 2013), masked } \\
\text { using AAL definition of vmPFC from } \\
\text { same reference }\end{array}$ & $-1,44,-7$ \\
\hline mPFC & $\begin{array}{l}10 \mathrm{~mm} \text { sphere around peak activation } \\
\text { in a preference construction task } \\
\text { (Barron et al., 2013) }\end{array}$ & $-6,53,13$ \\
\hline HPC & $\begin{array}{l}\text { Left and right hippocampal AAL masks } \\
\text { (Tzourio-Mazoyer et al., 2002) }\end{array}$ & \\
\hline Striatum & $\begin{array}{l}\text { Left and right putamen and caudate } \\
\text { AAL masks }\end{array}$ & \\
\hline aVLPFC & $\begin{array}{l}\text { 10 mm sphere around peak activation } \\
\text { in semantic retrieval task (Badre et al., } \\
\text { 2005a) }\end{array}$ & $-54,27,-9$ \\
\hline MTC & $\begin{array}{l}\text { 10 mm sphere around peak activation } \\
\text { in semantic classification task } \\
\text { (Thompson-Schill et al., 1997) }\end{array}$ & $-52,-55,2$ \\
\hline
\end{tabular}


1476 Table 2. fMRI activations for main effects of task conditions shown in Figure 4

\begin{tabular}{|c|c|c|c|c|c|}
\hline \multirow[t]{2}{*}{ Region (AAL2) } & \multicolumn{3}{|c|}{$\begin{array}{l}\text { MNI } \\
\text { Coordinates }\end{array}$} & \multirow[t]{2}{*}{$\begin{array}{l}\text { Number } \\
\text { of voxels }\end{array}$} & \multirow[t]{2}{*}{$\begin{array}{l}\text { Peak } t \text { - } \\
\text { value }\end{array}$} \\
\hline & $\mathrm{x}$ & $\mathrm{y}$ & $\mathrm{Z}$ & & \\
\hline \multicolumn{6}{|l|}{$\begin{array}{l}\text { Recipe }>\text { Independent, correct } \\
(\mathrm{k}=148)\end{array}$} \\
\hline Left superior frontal gyrus, medial orbital & -10 & 44 & -12 & 240 & 4.31 \\
\hline \multicolumn{6}{|l|}{$\begin{array}{l}\text { Positive }>\text { Negative Value, correct } \\
(\mathrm{k}=157)\end{array}$} \\
\hline Right anterior cingulate & 12 & 40 & 8 & 4134 & 7.60 \\
\hline Left superior frontal gyrus, medial & -10 & 54 & 14 & & 7.54 \\
\hline Right superior frontal gyrus, medial orbital & 4 & 34 & -14 & & 5.84 \\
\hline Right posterior orbital gyrus & 24 & 26 & -14 & 282 & 5.78 \\
\hline Right caudate nucleus & 6 & 12 & -4 & 172 & 4.66 \\
\hline \multicolumn{6}{|l|}{$\begin{array}{l}\text { Presentation } 1>2>3 \\
(\mathrm{k}=173)\end{array}$} \\
\hline Left inferior frontal gyrus, opercularis & -42 & 4 & 28 & 8816 & 12.39 \\
\hline Left supplementary motor area & -6 & 22 & 46 & & 11.67 \\
\hline Left inferior frontal gyrus, triangularis & -46 & 24 & 16 & & 11.30 \\
\hline Left inferior temporal gyrus & -48 & -52 & -16 & 4672 & 11.83 \\
\hline Left fusiform gyrus & -22 & -86 & -12 & & 8.76 \\
\hline Left fusiform gyrus & -36 & -70 & -12 & & 8.59 \\
\hline Right fusiform gyrus & 36 & -58 & -12 & 3287 & 10.21 \\
\hline Right inferior occipital gyrus & 34 & -88 & -6 & & 8.51 \\
\hline Right fusiform gyrus & 36 & -28 & -28 & & 6.24 \\
\hline Right middle frontal gyrus & 50 & 32 & 22 & 578 & 8.27 \\
\hline Left middle occipital gyrus & -24 & -68 & 36 & & 7.59 \\
\hline Left superior parietal gyrus & -30 & -62 & 66 & & 4.14 \\
\hline Right inferior frontal gyrus, orbital & 32 & 30 & -10 & 621 & 6.91 \\
\hline Left thalamus & -8 & -8 & 4 & 370 & 5.36 \\
\hline \multicolumn{6}{|l|}{$\begin{array}{l}\text { Correct > error } \\
(\mathrm{k}=210)\end{array}$} \\
\hline Right pallidum & 26 & -6 & -6 & 4394 & 8.87 \\
\hline Right superior temporal gyrus & 56 & -26 & 4 & 4394 & 6.39 \\
\hline Right middle temporal gyrus & 62 & -2 & -18 & 4394 & 6.13 \\
\hline Right paracentral lobule & 10 & -26 & 68 & 1065 & 5.29 \\
\hline Left precentral lobule & -22 & -22 & 60 & 1065 & 4.61 \\
\hline Left middle temporal gyrus & -46 & 2 & -28 & 6667 & 8.37 \\
\hline Left hippocampus & -24 & -10 & -22 & 6667 & 7.70 \\
\hline Left putamen & -30 & -2 & 2 & 6667 & 7.68 \\
\hline Right superior occipital gyrus & 24 & -90 & 30 & 1195 & 7.21 \\
\hline
\end{tabular}


bioRxiv preprint doi: https://doi.org/10.1101/712661; this version posted April 22, 2020. The copyright holder for this preprint (which was not certified by peer review) is the author/funder, who has granted bioRxiv a license to display the preprint in perpetuity. It is made available under aCC-BY-NC-ND 4.0 International license.

\begin{tabular}{|l|l|l|l|l|l|}
\hline Left cuneus & -8 & -94 & 22 & 1195 & 5.31 \\
\hline Left anterior cingulate & -2 & 28 & -8 & 241 & 4.54 \\
\hline Right superior frontal gyrus, medial & 6 & 50 & 0 & 241 & 3.79 \\
\hline
\end{tabular}

1477 All reported clusters were significant at the $P<0.05$, FWE-corrected threshold at the 1478 cluster level after peak thresholding at $P<0.001$. The critical cluster extent threshold for 1479 each contrast is given by the value of $\mathrm{k}$. 
bioRxiv preprint doi: https://doi org/10.1101/712661· this version posted April 22, 2020. The copyright holder for this preprint (which was not certified by peer review) is the author/funder, who has granted bioRxiv a license to display the preprint in perpetuity. It is made available under aCC-BY-NC-ND 4.0 International license.

1481 Table 3. Mean parameter values and Pearson correlations between parameters in the 1482 best-fitting behavioral model

\begin{tabular}{|l|l|l|l|l|l|l|l|}
\hline & $\begin{array}{l}\text { Mean } \\
(\text { SD) }\end{array}$ & Decay & $\begin{array}{l}\text { EV } \\
\text { temp. }\end{array}$ & $\begin{array}{l}\text { SV } \\
\text { temp. }\end{array}$ & UF temp. & $\begin{array}{l}\text { Fixed } \\
\text { bias }\end{array}$ & $\begin{array}{l}\text { Schema } \\
\text { discount } \\
\text { (log-space) }\end{array}$ \\
\hline Decay & $\begin{array}{l}\mathbf{0 . 0 2} \\
\mathbf{( 0 . 0 1 )}\end{array}$ & - & & & & & \\
\hline EV temp. & $\begin{array}{l}\mathbf{5 . 1 8} \\
(\mathbf{0 . 3 1})\end{array}$ & 0.28 & - & & & & \\
\hline SV temp. & $\begin{array}{l}\mathbf{2 . 4 5} \\
(\mathbf{1 . 7 0})\end{array}$ & 0.04 & 0.37 & - & & & \\
\hline UF temp. & $\begin{array}{l}\mathbf{- 0 . 8 4} \\
\mathbf{( 0 . 9 7 )}\end{array}$ & 0.12 & 0.27 & 0.21 & - & & \\
\hline Fixed bias & $\begin{array}{l}\mathbf{0 . 6 9} \\
\mathbf{( 0 . 7 0 )}\end{array}$ & 0.06 & -0.29 & -0.29 & $-0.94^{* * *}$ & - & \\
\hline $\begin{array}{l}\text { Schema } \\
\text { discount } \\
\text { (log-space) }\end{array}$ & $\begin{array}{l}\mathbf{- 2 . 4 7} \\
\mathbf{( 6 . 0 5 )}\end{array}$ & -0.20 & 0.28 & 0.15 & -0.03 & -0.06 & - \\
\hline $\begin{array}{l}\text { Recipe } \\
\text { dimensions } \\
\text { (log-space) }\end{array}$ & $\begin{array}{l}\mathbf{1 . 1 0} \\
\mathbf{( 0 . 3 6 )}\end{array}$ & -0.12 & -0.05 & 0.40 & -0.37 & 0.24 & 0.36 \\
\hline
\end{tabular}


bioRxiv preprint doi: https://doi.org/10.1101/712661; this version posted April 22, 2020. The copyright holder for this preprint (which was not certified by peer review) is the author/funder, who has granted bioRxiv a license to display the preprint in perpetuity. It is made available under aCC-BY-NC-ND 4.0 International license.

1485 Table 4. fMRI activations for model-based values GLM, including those from Figure 8

\begin{tabular}{|c|c|c|c|c|c|}
\hline \multirow[t]{2}{*}{ Region (AAL2) } & \multicolumn{3}{|c|}{ MNI Coordinates } & \multirow{2}{*}{$\begin{array}{l}\text { Number } \\
\text { of voxels }\end{array}$} & \multirow{2}{*}{$\begin{array}{l}\text { Peak } t- \\
\text { value }\end{array}$} \\
\hline & $\mathrm{X}$ & $\mathrm{y}$ & $\mathrm{z}$ & & \\
\hline \multicolumn{6}{|l|}{$\begin{array}{l}\text { Unfamiliarity } \\
(\mathrm{k}=161)\end{array}$} \\
\hline Left fusiform gyrus & -36 & -44 & -20 & 5443 & 11.02 \\
\hline Left inferior occipital gyrus & -28 & -84 & -10 & 5443 & 9.20 \\
\hline Left middle temporal gyrus & -60 & -48 & -8 & 5443 & 7.03 \\
\hline Right fusiform gyrus & 34 & -52 & -12 & 3877 & 9.98 \\
\hline Right lingual gyrus & 24 & -88 & -6 & 3877 & 9.50 \\
\hline Right fusiform gyrus & 34 & -28 & -28 & 3877 & 7.42 \\
\hline Left inferior frontal gyrus, orbitalis & -40 & 32 & -12 & 2995 & 9.54 \\
\hline $\begin{array}{l}\text { Left inferior frontal gyrus, } \\
\text { opercularis }\end{array}$ & -40 & 4 & 28 & 2995 & 9.17 \\
\hline $\begin{array}{l}\text { Left inferior frontal gyrus, } \\
\text { triangularis }\end{array}$ & -42 & 24 & 14 & 2995 & 8.45 \\
\hline $\begin{array}{l}\text { Left superior frontal gyrus, } \\
\text { dorsolateral }\end{array}$ & -16 & 38 & 50 & 1843 & 7.67 \\
\hline Left middle frontal gyrus & -32 & 18 & 58 & 1843 & 5.32 \\
\hline Left supplementary motor area & -6 & 12 & 56 & 1843 & 7.15 \\
\hline Left gyrus rectus & -2 & 48 & -18 & 403 & 7.08 \\
\hline Left precuneus & -4 & -54 & 18 & 173 & 6.80 \\
\hline Left hippocampus & -18 & -8 & -20 & 173 & 6.31 \\
\hline Right gyrus rectus & 2 & 28 & -20 & 173 & 3.94 \\
\hline \multicolumn{6}{|l|}{$\begin{array}{l}\text { Confidence } \\
(\mathrm{k}=158)\end{array}$} \\
\hline Left superior frontal gyrus, medial & -6 & 50 & 46 & 240 & 5.84 \\
\hline Left superior frontal gyrus, medial & -4 & 56 & 22 & 240 & 4.01 \\
\hline Left middle temporal gyrus & -58 & -12 & -16 & 187 & 4.25 \\
\hline Left middle temporal gyrus & -64 & -30 & 0 & 159 & 4.87 \\
\hline Right Postcentral gyrus & 24 & -32 & 64 & 196 & 4.58 \\
\hline \multicolumn{6}{|l|}{$\begin{array}{l}\text { Schema value } x \text { participant-P(yes) } \\
(\mathrm{k}=161)\end{array}$} \\
\hline Right middle temporal gyrus & 52 & -42 & 4 & 559 & 5.789 \\
\hline Right inferior temporal gyrus & 58 & -60 & -14 & 559 & 4.243 \\
\hline Right inferior frontal gyrus & 48 & 36 & 0 & 229 & 5.664 \\
\hline Left superior temporal gyrus & -54 & -6 & -2 & 256 & 5.168 \\
\hline Left middle temporal gyrus & -58 & 4 & -22 & 256 & 3.93 \\
\hline Right superior temporal gyrus & 66 & -10 & 2 & 269 & 5.015 \\
\hline Left cuneus & 2 & -82 & 38 & 301 & 4.872 \\
\hline Right cuneus & 18 & -86 & 26 & 301 & 3.882 \\
\hline
\end{tabular}


bioRxiv preprint doi: https://doi.org/10.1101/712661; this version posted April 22, 2020. The copyright holder for this preprint (which was not certified by peer review) is the author/funder, who has granted bioRxiv a license to display the preprint in perpetuity. It is made available under aCC-BY-NC-ND 4.0 International license.

\begin{tabular}{|l|l|l|l|l|l|}
\hline Left middle temporal gyrus & -66 & -50 & 2 & 250 & 4.747 \\
\hline Right supramarginal gyrus & 58 & -26 & 20 & 182 & 4.682 \\
\hline Left superior temporal gyrus & -64 & -30 & 18 & 193 & 4.657 \\
\hline $\begin{array}{l}\text { Experienced value x participant- } \\
\text { P(yes) } \\
(\mathrm{k}=168)\end{array}$ & & & & & \\
\hline Left superior frontal gyrus, medial & -4 & 60 & 8 & 1198 & 6.92 \\
\hline Left gyrus rectus & -8 & 38 & -16 & 1198 & 4.95 \\
\hline Right superior frontal gyrus, medial & 10 & 58 & 28 & 1198 & 4.18 \\
\hline Left angular gyrus & -50 & -76 & 24 & 185 & 5.21 \\
\hline
\end{tabular}

1486 All reported clusters were significant at the $P<0.05$, FWE-corrected threshold at the 1487 cluster level after peak thresholding at $P<0.001$. The critical cluster extent threshold for 1488 each contrast is given by the value of $\mathrm{k}$. 
1490 Table 5. Whole brain seed-to-voxel connectivity effects from Figure 10

\begin{tabular}{|c|c|c|c|c|c|}
\hline \multirow[t]{2}{*}{ Region (AAL2) } & \multicolumn{3}{|c|}{$\begin{array}{l}\text { MNI } \\
\text { Coordinates }\end{array}$} & \multirow[t]{2}{*}{$\begin{array}{l}\text { Number } \\
\text { of voxels }\end{array}$} & \multirow[t]{2}{*}{$\begin{array}{l}\text { Peak } t \text { - } \\
\text { value }\end{array}$} \\
\hline & $\mathrm{x}$ & $\mathrm{y}$ & $\mathrm{z}$ & & \\
\hline $\begin{array}{l}\text { Recipe Positive }>\text { Recipe negative, } \\
\text { presentation 1, correct }- \text { MTC seed } \\
(k=96)\end{array}$ & & & & & \\
\hline Left superior frontal gyrus, medial orbital & -2 & 46 & -12 & 351 & 6.42 \\
\hline Left middle temporal gyrus & -52 & -68 & 12 & 112 & 5.30 \\
\hline $\begin{array}{l}\text { Correct }>\text { Error, independent } \\
\text { presentations } 2 \text { and } 3 \text {, correct }- \text { HPC seed } \\
(\mathrm{k}=83)\end{array}$ & & & & & \\
\hline Left middle frontal gyrus & -40 & 52 & 20 & 253 & 6.14 \\
\hline Left superior frontal gyrus, dorsolateral & -18 & 54 & -4 & 253 & 5.66 \\
\hline Right insula & 36 & 18 & 0 & 100 & 5.76 \\
\hline Right superior frontal gyrus, dorsolateral & 30 & 54 & 14 & 122 & 5.76 \\
\hline
\end{tabular}

1491 All reported clusters were significant at the $P<0.05$, FWE-corrected threshold at the 1492 cluster level after peak thresholding at $P<0.001$. The critical cluster extent threshold for each contrast is given by the value of $\mathrm{k}$. 